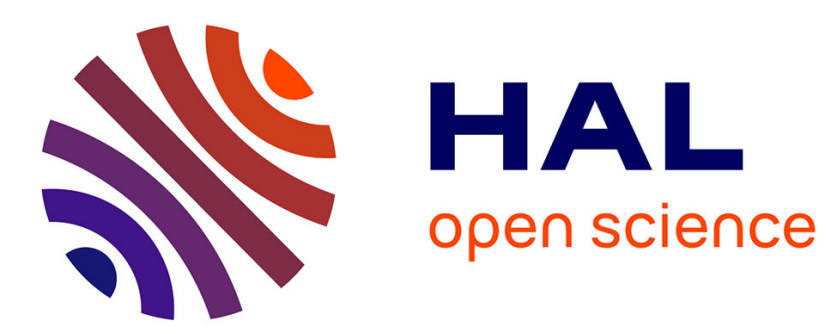

\title{
Full-field elastic simulations for image-based heterogeneous structures with a Coarse Mesh Condensation Multiscale Method
}

Minh Vuong Le, Julien Yvonnet, Nicolas Feld, Fabrice Detrez

\section{To cite this version:}

Minh Vuong Le, Julien Yvonnet, Nicolas Feld, Fabrice Detrez. Full-field elastic simulations for imagebased heterogeneous structures with a Coarse Mesh Condensation Multiscale Method. International Journal for Multiscale Computational Engineering, 2020, 18 (3), pp.305-327. hal-02618234

\section{HAL Id: hal-02618234 \\ https://hal.science/hal-02618234}

Submitted on 25 May 2020

HAL is a multi-disciplinary open access archive for the deposit and dissemination of scientific research documents, whether they are published or not. The documents may come from teaching and research institutions in France or abroad, or from public or private research centers.
L'archive ouverte pluridisciplinaire HAL, est destinée au dépôt et à la diffusion de documents scientifiques de niveau recherche, publiés ou non, émanant des établissements d'enseignement et de recherche français ou étrangers, des laboratoires publics ou privés. 


\title{
Full-field elastic simulations for image-based heterogeneous structures with a Coarse Mesh Condensation Multiscale Method
}

\author{
Minh Vuong Le ${ }^{\mathrm{a}, \mathrm{b}}$, Julien Yvonnet, ${ }^{\mathrm{a}, *}$, Nicolas Feld ${ }^{\mathrm{b}}$, Fabrice Detrez ${ }^{\mathrm{a}}$ \\ ${ }^{a}$ Univ Gustave Eiffel, CNRS, MSME UMR 8208, F-77454 Marne-la-Vallée, France \\ ${ }^{b}$ Safran Tech, Etablissement Paris Saclay, rue des Jeunes Bois-Châteaufort, 78114 Magny-les-Hameaux, France
}

\begin{abstract}
Micro tomography images allow obtaining fully detailed microstructural descriptions of heterogeneous materials and structures. To evaluate the effects of local gradients induced by the boundary conditions, it might be of interest to perform Direct Numerical Simulations (DNS) of such structures. In this paper, a multiscale method is developed to perform DNS on large, non-periodic linear heterogeneous structures with arbitrary boundary conditions and which can be performed in a classical Finite Element context. The method uses off-line calculations on subdomains that do not require to be periodic. Then, direct segmented images of the full 3D structure can be used directly without simplification. The novelty here is the use of non-periodic subdomains to decompose non-periodic heterogeneous structures and the possibility to use a coarse mesh which does not conform to the boundaries of the subdomains. As a result, the full-field finite element problem can be solved on the basis of the coarse mesh only, reducing drastically the computational costs. The accuracy of the method is analyzed on benchmarks and applications on large heterogeneous structures such as arising from 3D microtomography images are presented.
\end{abstract}

Keywords: Domain Decomposition, Composites, Multiscale methods, CMCM, Heterogeneous Structures, Tomography Images

\section{Introduction}

Direct Numerical Simulation (DNS) of heterogeneous structures, i.e. including an explicit description of all heterogeneities in the whole structure is unreachable when scales are separated, i.e. when the size of heterogeneities is small as compared to the size of the structure. However, weakly separated scales can occur in some cases: (i) for composites with large heterogeneities such as in woven composites, where the size of the strands is not negligible as compared to the size of the whole part; (ii) in some experimental samples which are small in size for practical reasons, as e.g. in samples studies in X-ray micro CT analysis. Engineers may be interested in performing DNS either to perform local field analysis or studying the stress concentration around a defect. In the mentioned cases, even though the number of heterogeneities can remain extremely large, the size of the problem may be reachable with direct solvers in some situations. The advantage of such analyses is to overcome the spurious effects of boundaries, global gradients, concentrated loads and randomness which are not well reproduced by homogenization techniques in the case of weakly separated scales, even though many approaches have been proposed to treat this problem, including: gradient-based homogenization techniques [1, 2, 3, 4, 5, 6], asymptotic expansion-based homogenization [7], higher order strain gradient homogenization [8], nonlocal homogenization [9], or computational continua method [10, 11]. Error estimators for activating RVE calculations in regions of the structure of high strain gradients and where uniform strains are no longer valid in computational homogenization have been proposed by Fish et al. in [12].

To perform DNS simulations of large heterogeneous structures, one possible strategy is parallel computing based on domain decomposition methods, including Finite Element Tearing and Interconnecting (FETI) [13] and Balanced

\footnotetext{
${ }^{*}$ Corresponding author: J. Yvonnet, Univ Gustave Eiffel, CNRS, MSME UMR 8208, F-77454 Marne-la-Vallée, France. E-mail: julien.yvonnet@univ-paris-est.fr
} 
Domain Decomposition (BDD) [14]. The special case of heterogeneous domains induces issues in these approaches, and more specifically convergence problems when the boundaries of the subdomains are cut but interfaces and for high contrasts of mechanical properties. To alleviate this issue, FETI and BDD have been extended to heterogeneous structures using preconditioners [15] and initialization [16]. However when dealing with structures containing large heterogeneities and high contrast of phase properties, these methods often exhibit poor performance. Several techniques have been proposed to alleviate these issues such as FETI-Geneo (FETI-generalized eigenvalues in the overlaps) [17], Multipreconditioned FETI method (MPFETI) [18] or Adaptive MultiPreconditioned FETI (AMPFETI) [19, 20]. Another technique, the Large Time Increment (LATIN) method [21, 22], is a non-incremental iterative computational strategy where local (nonlinear) problems solutions in subdomains are updated through a global linear problem through interfaces conditions and an appropriate search direction to ensure the global convergence. In addition, the search directions are computed using the principles of homogenization.

Another parallel HPC strategy is the algebraic multigrid method (AMG) [23, 24, 25, 26], whose aim is to directly solve the large linear systems of equations obtained by FEM discretizations, by coarsening the linear system of equations using a coarse grid in order to reduce the problem size.

In recent years, multiscale methods have been developed to solve heterogeneous material problems and can be derived to solve heterogeneous structures. Many approaches have been included, as e.g. Zohdi's method [27, 28], multiscale methods or subdomain methods with appropriate reduction [29, 30, 31, 32], the Multiscale Finite Element method [33, 34], or micro-macro subdomain coupling techniques [35, 36, 37]. These approaches are able to solve heterogeneous structures, but still present some limitations. For example, in Zhodi's method [27, 28], in order to solve the problem, scale separation hypothesis needs to be considered, wich essentially means that the characteristic length of the heterogeneities need to be much smaller than those of the macro structure. In the method proposed by Hou et al. [33, 34], there exists discontinuities in the macro solutions, which causes difficulties in the relocalization process. In addition to the above methods, in [38], the authors have proposed a variational segmentation approach that computes the macro scale problem based on a prior optimized X-ray microtomography model at a smaller scale. The micro information in each macro element, including gray levels and local directions, are determined by overlapping the macro mesh and the X-ray microtomography model. This approach is able to tackle complex woven composites, however requires a preprocessing step in order to generate the prior model. The more complex the material is, the more expensive this step gets. In [39], the authors have proposed a technique of non-intrusive surface coupling for the global-local analysis of heterogeneous structures. This approach consists in studying specifically the response of a detailed zone of interest inside the structure. The linkage between the micro and macro scales are determined using a second order gradient kinematic for the interface displacements. Finally, a combination of homogenization principles and multigrid methods for an efficient solution of heterogeneous structures has been considered in [40, 41, 42, 43].

Another very popular method to perform DNS on image-based models of microstructures is the Fast Fourier Transform (FFT) method, initially proposed by Moulinec and Suquet [44]. The method uses a matrix-free iterative solver on regular grids, can be efficiently parallelized [45] and has proved to be much more efficient than FEM in various cases [46]. However, FFT is dedicated to periodic microstructures and periodic boundary conditions, and its applicability to non-cubic domains/arbitrary boundary conditions (including Dirichlet and Neumann) is so far an open issue, even though an example of applicability of FFT on tubular structures can be found in [45].

In this work, a Coarse mesh Condensation Multiscale (CMCM) method, initially introduced in [47] for solving periodic composite structures, is extended here to perform direct numerical simulations (DNS) of large, non-periodic heterogeneous structures as found e.g. in micro tomography image-based models. The main idea of the method is to use a reduced representation of the subdomains boundary displacements, which can be employed to condense the solutions in the subdomains on their boundaries. Then, the reduced degrees of freedom (dof) are expressed with respect to the dof of a mapping coarse mesh on the whole structure. As a result, the problem is solved on the coarse mesh dof only. The first novelty introduced in this paper is the use of non-periodic subdomains which allows decomposing arbitrary, non-periodic heterogeneous structures. The second novelty is the use of a non-conforming coarse mesh. Then, it is no more required that the coarse mesh conforms with the subdomain boundaries and can then use the benefit of local refinement of the coarse mesh around singularities to improve the accuracy.

The paper is organized as follows. In section 3, the basic CMCM is reviewed. In section 4, its extensions to non-periodic meshes are presented, including an enhanced integration method for fine scales elements cut by coarse mesh elements. Finally, numerical examples are presented to discuss the error analysis in severe cases such as when heterogeneities cut the subdomains boundaries, effect of local refinement of the coarse mesh around singularities. 
Finally, the potential of the method to perform DNS on 3D micro tomography models of heterogeneous structures directly involving hundreds of millions of dof on a standard computer, is shown.

\section{Preliminary: notations}

\begin{tabular}{rll}
\hline $\mathbf{x}$ & $:$ & Position vector at the fine scale \\
$\mathbf{u}(\mathbf{x}) \quad:$ & Displacement vector at the fine scale \\
$\boldsymbol{\varepsilon}$ & $:$ & Linearized strain tensor \\
$\boldsymbol{\sigma}$ & $:$ & Cauchy stress tensor \\
$\mathbb{C}(\mathbf{x}) \quad:$ & Elastic tensor \\
$\overline{\boldsymbol{\varepsilon}}$ & $:$ & Macroscopic linearized strain tensor \\
$\overline{\mathbf{g}}^{\alpha}$ & $:$ & Vector of parameters defining Dirichlet boundary conditions on the \\
& & boundary of $\Omega^{\alpha}$ \\
$\Omega^{\alpha}$ & $:$ & Subdomain $\alpha$ \\
$\mathbf{A}^{\alpha}(\mathbf{x})$ & $:$ & Localization tensor in $\Omega^{\alpha}$ \\
{$[\boldsymbol{\varepsilon}(\mathbf{x})]$} & $:$ & Vector for associated with $\boldsymbol{\varepsilon}(\mathbf{x})$ \\
{$[\boldsymbol{\sigma}(\mathbf{x})]$} & $:$ & Vector form associated with $\boldsymbol{\sigma}(\mathbf{x})$ \\
$\mathbf{C}(\mathbf{x})$ & $:$ & Matrix form associated with $\mathbb{C}(\mathbf{x})$ \\
$\Omega^{e}$ & $:$ & Domain associated with an element $e$ in the fine mesh \\
$\Omega^{E}$ & $:$ & Domain associated with an element $E$ in the coarse mesh \\
$\mathbf{u}^{e}$ & $:$ & Vector of nodal displacements in one element $e$ of the fine mesh \\
$\overline{\mathbf{u}}^{E}$ & $:$ & Vector of nodal displacements in one element $E$ of the coarse mesh \\
$N d^{E}$ & $:$ & Number of degrees of freedom of a macro element $E$ \\
\hline & &
\end{tabular}

Table 1: Notations

\section{Coarse Mesh Condensation Multiscale (CMCM) Method for periodic subdomains}

In the present work, the objective is to perform FEM elastic simulations on realistic geometrical models of microstructures directly obtained from X-Ray micro computed tomography (XR- $\mu \mathrm{CT}$ ). For this purpose, finite elements meshes must be constructed from the segmented images, which are obtained by applying filters or morphological operators on the grey-level initial images. Details about such techniques can be found e.g. in [48]. Then, two techniques can be employed to construct the FEM meshes. The first one consists in converting each voxel of the segmented image into a cubic domain, which can be associated with one or several 8-node elements or which can be decomposed into tetrahedra. Then, the properties defined in each phase from the segmented image are mapped onto the cubic domains. The advantages of this strategy are its simplicity and the possibility to use parallel solving procedures related to regular meshes in the numerical simulations. The main drawback comes from the obtained large meshes. Adaptive mesh refinement is possible in such regular meshes through e.g. octree meshes [49]. Accuracy of voxel-based models has been studied e.g. in [50]. The second strategy is to use a meshing software able to work on initial data in the form of 3D voxel-based segmented image, and which is able to reconstruct and mesh the interfaces. Several meshing codes are available, like Cgal $($, Tetgen $\AA$, Iso2mesh $囚$, or AVIZOß. Examples of constructions of such unstructured meshes can be found in [51]. In this paper, we will use the first mentioned technique for large applications, i.e. using regular meshes matching the voxel data, while the second technique (structured meshes) will be applied for the studied benchmark problems.

We consider a heterogeneous structure defined in a domain $\Omega \subset \mathbb{R}^{D}, D=2,3$, with boundary $\partial \Omega=\partial \Omega_{u} \cup \partial \Omega_{F}$, $\partial \Omega_{u} \cap \Omega_{F}=\emptyset$, where $\partial \Omega_{u}$ and $\partial \Omega_{F}$ denote displacement (Dirichlet) and traction (Neumann) boundaries (see Fig. 2 (a)). The objective of CMCM is to provide an approximation of the full-field solution in the heterogeneous structure when scale separation assumption does not hold, through a parallel solving procedure. The different steps of the method are described below. 

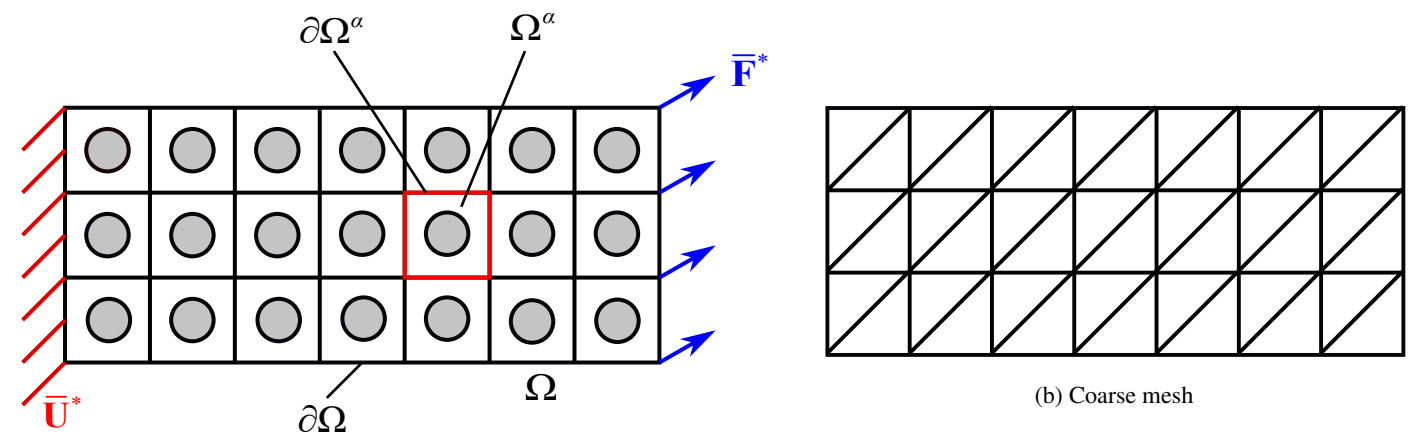

(b) Coarse mesh

(a) Decomposition of the periodic structure into subdomains

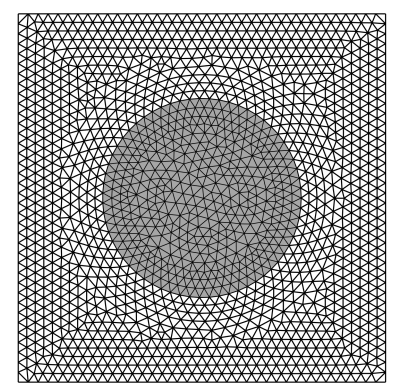

(c) Subdomain $\Omega^{\alpha}$ and related fine mesh

Figure 1: CMCM for periodic structures.

\subsection{Parallel solving of local problems}

The first step of the method is to decompose the structure into $N$ subdomains $\Omega^{\alpha}, \alpha=1,2, \ldots, N$ such that

$$
\Omega=\bigcup_{\alpha=1}^{N} \Omega^{\alpha}
$$

(see Fig. 2(a)). The boundaries of $\Omega^{\alpha}$ are denoted by $\partial \Omega^{\alpha}$. Then, each subdomain is meshed at the scale of the heterogeneities. This mesh is denoted in the following as the fine mesh. Next, we define elastic problems to be solved independently over each subdomain. For this purpose, Dirichlet boundary conditions are prescribed over $\partial \Omega^{\alpha}$ as:

$$
\mathbf{u}(\mathbf{x})=\mathbf{D}(\mathbf{x}) \overline{\mathbf{g}}^{\alpha} \quad \text { on } \partial \Omega^{\alpha},
$$

where $\overline{\mathbf{g}}^{\alpha}$ is a vector of scalar parameters and $\mathbf{D}(\mathbf{x})$ is a matrix of functions depending on $\mathbf{x}$. For example, in this work, we use the following boundary conditions in $2 \mathrm{D}$ :

$$
\mathbf{u}(\mathbf{x})=\underbrace{\left[\begin{array}{ccc}
x_{1} & 0 & \frac{1}{2} x_{2} \\
0 & x_{2} & \frac{1}{2} x_{1}
\end{array}\right]}_{\mathbf{D}(\mathbf{x})} \underbrace{\left[\begin{array}{c}
\bar{\varepsilon}_{11}^{\alpha} \\
\bar{\varepsilon}_{22}^{\alpha} \\
2 \bar{\varepsilon}_{12}^{\alpha}
\end{array}\right]}_{\overline{\mathbf{g}}^{\alpha}},
$$



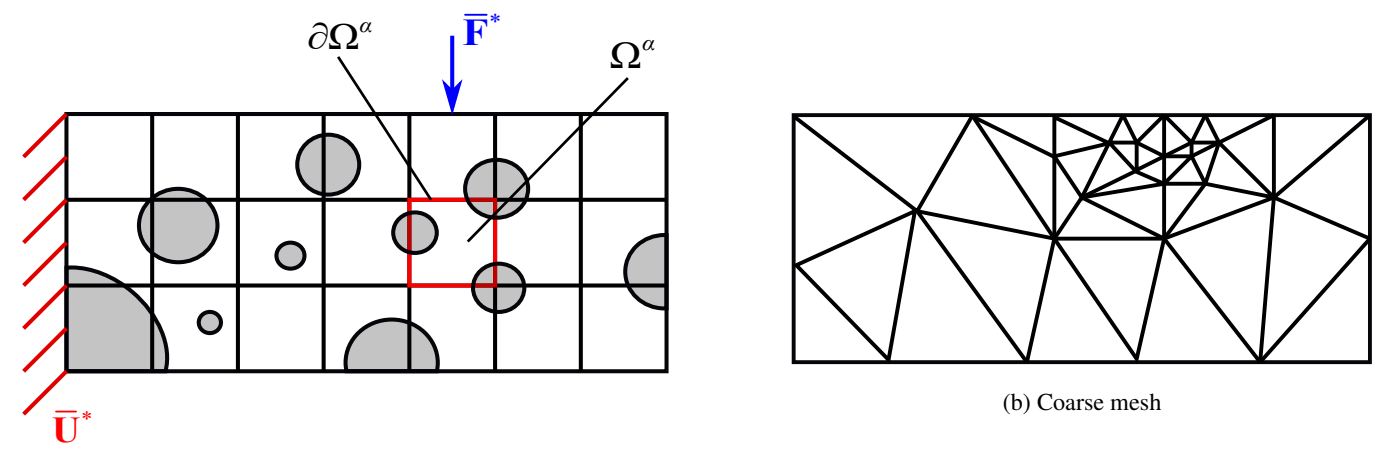

(b) Coarse mesh

(a) Decomposition of the non-periodic structure into subdomains

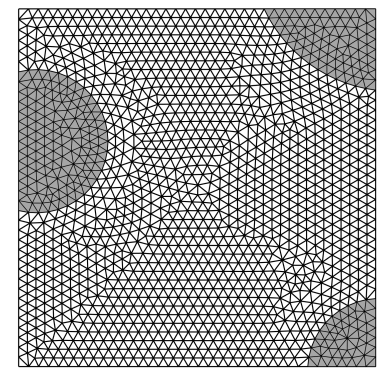

(c) Subdomain $\Omega^{\alpha}$ and related fine mesh

Figure 2: Unstructured CMCM for non-periodic structures.

and in 3D:

$$
\mathbf{u}(\mathbf{x})=\underbrace{\left[\begin{array}{llllll}
x_{1} & 0 & 0 & 0 & \frac{1}{2} x_{3} & \frac{1}{2} x_{2} \\
0 & x_{2} & 0 & \frac{1}{2} x_{3} & 0 & \frac{1}{2} x_{1} \\
0 & 0 & x_{3} & \frac{1}{2} x_{2} & \frac{1}{2} x_{1} & 0
\end{array}\right]}_{\mathbf{D}(\mathbf{x})} \underbrace{\left[\begin{array}{c}
\bar{\varepsilon}_{11}^{\alpha} \\
\bar{\varepsilon}_{22}^{\alpha} \\
\bar{\varepsilon}_{33}^{\alpha} \\
2 \bar{\varepsilon}_{23}^{\alpha} \\
2 \bar{\varepsilon}_{13}^{\alpha} \\
2 \bar{\varepsilon}_{12}^{\alpha}
\end{array}\right],}_{\overline{\mathbf{g}}^{\alpha}}
$$

which are identical to classical KUBC boundary conditions in homogenization (see e.g. [52]). Then, on each subdomain $\Omega^{\alpha}$, the following local problems are solved:

Given $\overline{\mathbf{g}}^{\alpha}$, find $\boldsymbol{\varepsilon}(\mathbf{x})$ in $\Omega^{\alpha}$ such that:

$$
\begin{gathered}
\nabla \cdot(\mathbb{C}(\mathbf{x}): \boldsymbol{\varepsilon}(\mathbf{x}))=0 \quad \forall \mathbf{x} \in \Omega^{\alpha}, \\
\mathbf{u}(\mathbf{x})=\mathbf{D}(\mathbf{x}) \overline{\mathbf{g}}^{\alpha} \quad \text { on } \partial \Omega^{\alpha}, \quad \alpha=1,2, \ldots, N .
\end{gathered}
$$

The problem being linear, we can express the local strain (on the fine mesh) of each elementary problem as:

$$
[\boldsymbol{\varepsilon}(\mathbf{x})]=\mathbf{A}^{\alpha}(\mathbf{x}) \overline{\mathbf{g}}^{\alpha} \quad \forall \mathbf{x} \in \Omega^{\alpha},
$$

where $\mathbf{A}^{\alpha}(\mathbf{x})$ is a localization matrix relating the local strain field in the fine mesh to the values in $\overline{\mathbf{g}}^{\alpha}$, which can be defined differently, e.g. by adding higher order terms [47] or other functions.

The number of local problems in each subdomain is equal to the length of $\overline{\mathbf{g}}^{\alpha}$, which is here 3 in $2 \mathrm{D}$ and 6 in $3 \mathrm{D}$. Therefore, we have $3 \times N$ and $6 \times N$ problems to be solved in $2 \mathrm{D}$ and $3 \mathrm{D}$, respectively. However, each of these problems is computed on a small part of the structure, and can thus be solved efficiently. On the other hand, all problems are 
independent and can be solved in parallel to reduce computational time. It can be noted that the computational time of local problems scales linearly with the number of available processors. Then, we can deduce that the computational time for solving all subdomains problems is $3 \times N / N_{p}$ in $2 \mathrm{D}$ and $6 \times N / N_{p}$ in $3 \mathrm{D}$ with $N_{p}$ is the number of processors. The solutions of the local problems are stored for the next step. In $2 \mathrm{D}$, the columns of $\mathbf{A}^{\alpha}(\mathbf{x})$ are formed by the solutions of each elementary problems as

$$
\mathbf{A}^{\alpha}(\mathbf{x})=\left[\left[\varepsilon^{1}(\mathbf{x})\right],\left[\varepsilon^{2}(\mathbf{x})\right],\left[\varepsilon^{3}(\mathbf{x})\right]\right],
$$

where $\left[\varepsilon^{i}(\mathbf{x})\right]$ is the strain vector solution of the local problem whose boundary conditions are defined associated with $\bar{g}_{i}^{\alpha}=1, g_{j}^{\alpha}=0, j \neq i$. In $3 \mathrm{D}, \mathbf{A}^{\alpha}(\mathbf{x})$ has then 6 columns. Then, $\mathbf{A}^{\alpha}(\mathbf{x})$ is a $3 \times 3$ matrix in $2 \mathrm{D}$ and a $6 \times 6$ matrix in $3 \mathrm{D}$.

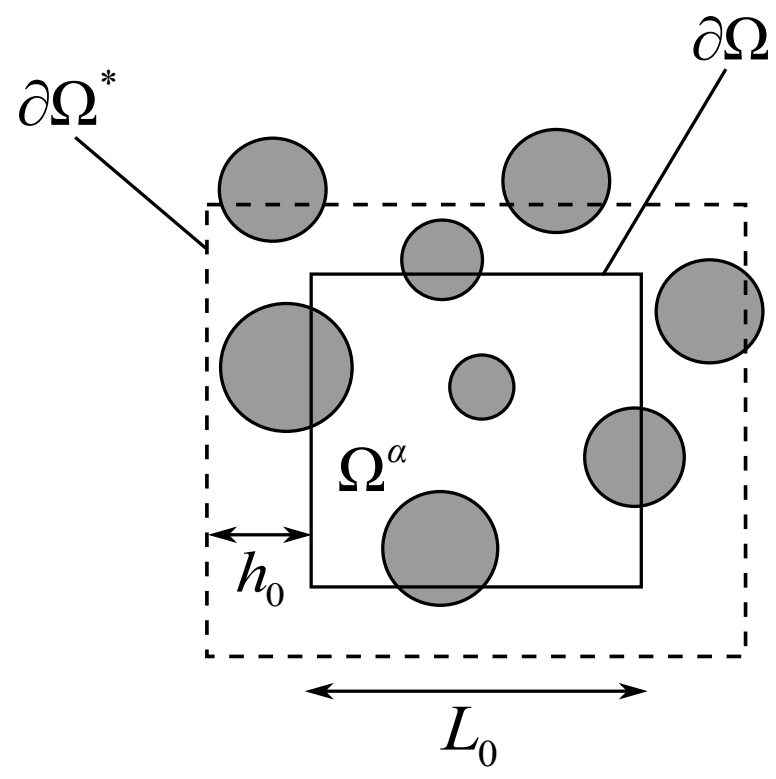

Figure 3: Extended subdomain illustration.

We have shown in [47] that when interfaces cross the boundaries of the subdomains $\Omega^{\alpha}$, large errors may be induced on the local fields. To overcome this issue, we have proposed in the same reference an extended subdomain technique, where the boundary conditions $(2)$ are prescribed on a larger boundary $\partial \Omega^{*}$ embedding $\Omega^{\alpha}$ (see Fig 3 ). In this extension, the matrix $\mathbf{A}^{\alpha}(\mathbf{x})$ is still stored only in the elements lying inside the original subdomain $\Omega^{\alpha}$. The size of the extended subdomain is defined by a parameter $\beta$ defined as

$$
\beta=\frac{L_{0}}{h_{0}}
$$

where $L_{0}$ is the length of the initial subdomain and $h_{0}$ is the thickness of the extended part.

\subsection{Coarse mesh condensation}

In the next step, the structure is now discretized over the whole domain $\Omega$ by a coarse mesh (see Fig. 2(b)). The coarse mesh does not necessarily conforms with the boundaries of the subdomains $\partial \Omega^{\alpha}$, i.e. elements of the fine mesh can intersect those of the coarse mesh (see Fig. 4).

Given nodal displacements $\overline{\mathbf{u}}^{e}$ in the coarse mesh, the strain field on the coarse mesh is defined by:

$$
[\overline{\boldsymbol{\varepsilon}}(\mathbf{x})]=\overline{\mathbf{B}}^{E}(\mathbf{x}) \overline{\mathbf{u}}^{E}
$$

where $\overline{\mathbf{B}}^{E}(\mathbf{x})$ is the finite element shape function derivatives of element $E$ defined on the coarse mesh. For a Gauss point $\mathbf{x}_{k}$ in $\Omega, \mathbf{x}_{k}$ belongs both to (i) a coarse mesh element $E$ and (ii) a fine mesh element $e$ lying in a subdomain $\Omega^{\alpha}$ (see Fig. 4). 


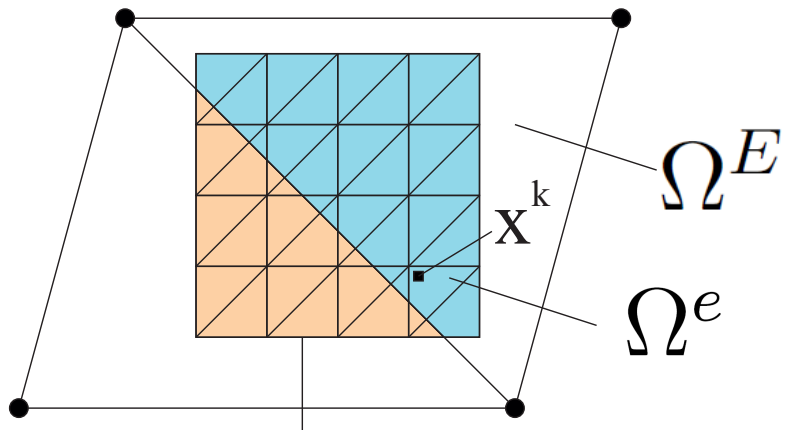

$\Omega^{\alpha}$

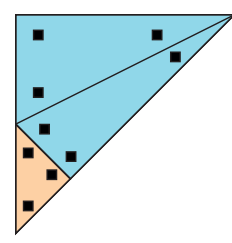

(b)

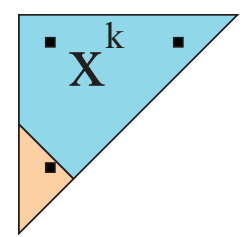

(c)

Figure 4: Subdomain $\Omega^{\alpha}$ cut by a coarse mesh element and integration subdomains indicated by different colors.

We define the distance in the sense of the $L_{2}$-norm between the strain field defined over the fine mesh $[\boldsymbol{\varepsilon}(\mathbf{x})]$ and the strain defined over each element of the coarse mesh $[\overline{\boldsymbol{\varepsilon}}(\mathbf{x})]$ as:

$$
J=\int_{\Omega^{E}}([\boldsymbol{\varepsilon}(\mathbf{x})]-[\overline{\boldsymbol{\varepsilon}}(\mathbf{x})])^{2} d \Omega
$$

where $(\mathbf{v})^{2}=\mathbf{v} \cdot \mathbf{v}$. Using (7), 10, and (1), we can write :

$$
J=\sum_{E} \int_{\Omega^{E}}\left(\mathbf{A}^{\alpha}(\mathbf{x}) \overline{\mathbf{g}}^{\alpha}-\overline{\mathbf{B}}^{E}(\mathbf{x}) \overline{\mathbf{u}}^{E}\right)^{2} d \Omega .
$$

The objective here is to minimize the distance of strain fields between micro and macro scale. For this purpose the minimization of $J$ needs to be performed. This process can be then done independently in each element of the coarse mesh:

$$
\overline{\mathbf{g}}^{\alpha}=\operatorname{Argmin} J^{\alpha}=\operatorname{Argmin} \int_{\Omega^{E}}\left(\mathbf{A}^{\alpha}(\mathbf{x}) \overline{\mathbf{g}}^{\alpha}-\overline{\mathbf{B}}^{E}(\mathbf{x}) \overline{\mathbf{u}}^{E}\right)^{2} d \Omega .
$$

We obtain a linear system in $\Omega^{\alpha}$ as:

$$
\underbrace{\int_{\Omega^{E}}\left[\left(\mathbf{A}^{\alpha}(\mathbf{x})\right)^{T} \mathbf{A}^{\alpha}(\mathbf{x})\right] d \Omega \overline{\mathbf{g}}^{\alpha}}_{\mathbf{H}^{\alpha}}=\underbrace{\int_{\Omega^{E}}\left(\mathbf{A}^{\alpha}(\mathbf{x})\right)^{T} \overline{\mathbf{B}}^{E}(\mathbf{x}) d \Omega}_{\mathbf{L}^{\alpha}} \overline{\mathbf{u}}^{E} .
$$

where the size of $\mathbf{H}^{\alpha}$ is $3 \times 3$ in $2 \mathrm{D}$ and $6 \times 6$ in $3 \mathrm{D}$; and the size of $L^{\alpha}$ is $3 \times N d^{E}$ in $2 \mathrm{D}$ and $6 \times N d^{E}$ in $3 \mathrm{D}$ with $N d^{E}$ is the number of degrees of freedom of a macro element $E$.

Then we can express $\overline{\mathbf{g}}^{\alpha}$ as a function of the coarse mesh nodal displacements as:

$$
\overline{\mathbf{g}}^{\alpha}=\left(\mathbf{H}^{\alpha}\right)^{-1} \mathbf{L}^{\alpha} \overline{\mathbf{u}}^{E}=\mathbf{W}^{\alpha} \overline{\mathbf{u}}^{e}
$$

with

$$
\mathbf{W}^{\alpha}=\left(\mathbf{H}^{\alpha}\right)^{-1} \mathbf{L}^{\alpha}
$$

Finally, using (7) and (15), a linear relationship between local strain in the fine mesh and the coarse mesh dofs can be established as:

$$
[\boldsymbol{\varepsilon}(\mathbf{x})]=\mathbf{T}^{\alpha}(\mathbf{x}) \overline{\mathbf{u}}^{E} \forall \mathbf{x} \in \Omega^{\alpha}
$$


with

$$
\mathbf{T}^{\alpha}(\mathbf{x})=\mathbf{A}^{\alpha}(\mathbf{x}) \mathbf{W}^{\alpha} .
$$

where $\mathbf{T}^{\alpha}(\mathbf{x})$ has the same size as $L^{\alpha}$, which is $3 \times N d^{E}$ in $2 \mathrm{D}$ and $6 \times N d^{E}$ in $3 \mathrm{D}$.

\subsection{Solving the problem on the coarse mesh}

At this step, we can note that the local strain (on the fine mesh) can be fully expressed as a function of the coarse mesh nodal displacements. The objective is then to express the problem to be solved on the coarse mesh. The full-field problem on the fine mesh is given by:

$$
\left\{\begin{array}{l}
\nabla \cdot \sigma(\mathbf{x})=0 \quad \forall \mathbf{x} \in \Omega, \\
\sigma(\mathbf{x})=\mathbb{C}(\mathbf{x}): \boldsymbol{\varepsilon}(\mathbf{x}) \forall \mathbf{x} \in \Omega, \\
\mathbf{u}(\mathbf{x})=\overline{\mathbf{u}}^{*} \forall \mathbf{x} \in \partial \Omega_{u} \\
\sigma \cdot \mathbf{n}=\overline{\mathbf{f}}^{*} \forall \mathbf{x} \in \partial \Omega_{f}
\end{array}\right.
$$

The corresponding weak form is given by, using vector forms for second-order tensors and matrix form for fourthorder tensor as:

$$
\int_{\Omega}[\varepsilon(\mathbf{u})]^{T} \mathbf{C}(\mathbf{x})[\varepsilon(\delta \mathbf{u})] d \Omega=\int_{\partial \Omega_{f}} \overline{\mathbf{f}}^{*} \cdot \delta \mathbf{u} d \Gamma .
$$

Introducing (17) in 23], we obtain:

$$
\delta \overline{\mathbf{u}}^{E^{T}} \sum_{\alpha} \int_{\Omega^{\alpha}}\left(\mathbf{T}^{\alpha}(\mathbf{x})\right)^{T} \mathbf{C}(\mathbf{x}) \mathbf{T}^{\alpha}(\mathbf{x}) d \Omega \overline{\mathbf{u}}^{E}=\delta \overline{\mathbf{u}}^{E^{T}} \int_{\partial \Omega_{f}} \overline{\mathbf{N}}^{T}(\mathbf{x}) \overline{\mathbf{f}}^{*} d \Gamma .
$$

Finally, owing to the arbitrariness of $\delta \mathbf{u}^{e}$, we obtain the linear system involving only the coarse mesh dofs as:

$$
\overline{\mathbf{K}} \overline{\mathbf{u}}=\overline{\mathbf{f}}
$$

where:

$$
\begin{gathered}
\overline{\mathbf{K}}=\sum_{\alpha} \int_{\Omega^{\alpha}}\left(\mathbf{T}^{\alpha}(\mathbf{x})\right)^{T} \mathbf{C}(\mathbf{x}) \mathbf{T}^{\alpha}(\mathbf{x}) d \Omega, \\
\overline{\mathbf{f}}=\int_{\partial \Omega_{f}} \overline{\mathbf{N}}^{T}(\mathbf{x}) \overline{\mathbf{f}}^{*} d \Gamma,
\end{gathered}
$$

and $\overline{\mathbf{N}}(\mathbf{x})$ are the classical finite element shape functions of elements in the coarse mesh. Once the global problem is solved, the local strain field of each subdomain can be reconstructed using (17) and the stress field is reconstructed using:

$$
[\boldsymbol{\sigma}(\mathbf{x})]=\mathbf{C}(\mathbf{x}) \mathbf{T}^{\alpha}(\mathbf{x}) \overline{\mathbf{u}}^{E}, \forall \mathbf{x} \in \Omega^{\alpha} .
$$

\section{Handling incompatible micro and coarse meshes}

Integration of $\mathbf{L}^{\alpha}$ in (14) requires special attention in the case when one subdomain is cut by the boundary of a coarse mesh element $\Omega^{E}$ (see Fig. 4). In that case, a consistent integration requires subdividing the cut micro elements $\Omega^{e}$ for integration purpose, as shown in Fig. 4(b). Denoting the set of micro elements by $\mathcal{S}_{0}$ and the set of subdivided micro elements by $\mathcal{S}_{1}$, a consistent integration of $\mathbf{L}^{\alpha}$ is expressed as:

$$
\mathbf{L}^{\alpha}=\sum_{e \in \mathcal{S}_{1}} \sum_{k}\left[\mathbf{A}^{\alpha}\left(\mathbf{x}_{k}\right)\right]^{T} \overline{\mathbf{B}}^{E}\left(\mathbf{x}_{k}\right) V^{e} w_{k},
$$

where $\mathbf{x}_{k}$ and $w_{k}$ denote the Gauss point positions and weights, respectively, and $V^{e}$ is the volume of the micro element in $\Omega^{e}$. In the present work, we used the Matlab@ command "intersect" to construct the set of subdivided elements. 


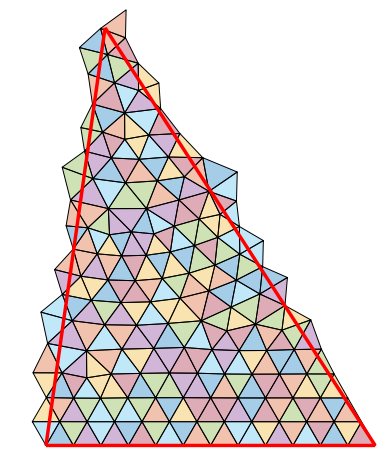

(a) Coarse mesh element $\Omega^{E}$ (in red) and micro elements contained within $\Omega^{E}$.

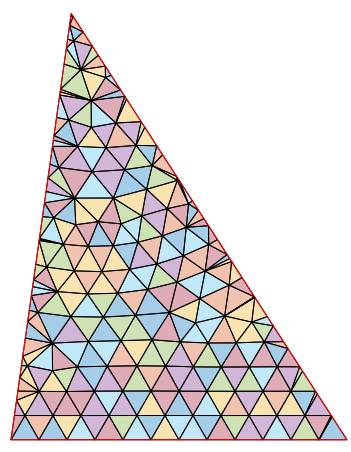

(b) Subdivision of micro elements contained within $\Omega^{E}$ for integration purpose.

Figure 5: Remeshing operation in the case where micro elements are cut by a macro element.

This algorithm returns the geometric intersection of 2 polygons as inputs. An illustration of this process is shown in Fig. 5 .

However, this operation can be costly and burdensome in the 3D case. Alternatively, an approximated integration scheme is proposed as

$$
\mathbf{L}^{\alpha} \simeq \sum_{e \in \mathcal{S}_{0}} \sum_{k}\left[\mathbf{A}^{\alpha}\left(\mathbf{x}_{k}\right)\right]^{T} \overline{\mathbf{B}}^{E}\left(\mathbf{x}_{k}\right) V^{e} w_{k},
$$

where $\mathcal{S}_{0}$ is the set of micro elements. In this integration scheme, the local values of $\mathbf{A}^{\alpha}(\mathbf{x})$ are affected according to the position of Gauss points in the micro elements (see Fig. 4(c)), but no subdivision of micro elements is performed for the sake of simplicity. Then here, the interface between two macro elements $\Omega^{E}$ is not explicitly described. The errors induced by this approximated integration scheme will be evaluated in the example section 5.2

\section{Numerical examples}

The objectives of the following numerical examples is to illustrate the capability of the method to handle: (a) non-uniform, possibly refined coarse meshes and (b) non-periodic microstructures, such as e.g. arising in micro tomography image based models.

In each example, two solutions are compared:

- A reference solution, denoted by "ref" in the different figures, is obtained by a Direct Numerical Simulation (DNS)- finite element calculation on a fully meshed structure at the scale of the heterogeneities.

- A solution obtained by the CMCM method.

For a better visualization of the results, we define a local error in the energy norm in one element $\Omega^{e}$ at the micro scale as:

$$
E R R^{e}=\frac{\int_{\Omega^{e}}\left(\boldsymbol{\varepsilon}^{r e f}(\mathbf{x})-\varepsilon^{C M C M}(\mathbf{x})\right): \mathbb{C}(\mathbf{x}):\left(\boldsymbol{\varepsilon}^{r e f}-\varepsilon^{C M C M}(\mathbf{x})\right) d \Omega^{e}}{\int_{\Omega^{e}} \boldsymbol{\varepsilon}^{r e f}: \mathbb{C}: \boldsymbol{\varepsilon}^{r e f} d \Omega^{e}}
$$

and a global error in the energy norm defined over the whole domain as:

$$
E R R=\frac{\int_{\Omega}\left(\boldsymbol{\varepsilon}^{r e f}(\mathbf{x})-\boldsymbol{\varepsilon}^{C M C M}(\mathbf{x})\right): \mathbb{C}(\mathbf{x}):\left(\boldsymbol{\varepsilon}^{r e f}(\mathbf{x})-\boldsymbol{\varepsilon}^{C M C M}(\mathbf{x})\right) d \Omega}{\int_{\Omega} \boldsymbol{\varepsilon}^{r e f}(\mathbf{x}): \mathbb{C}(\mathbf{x}): \boldsymbol{\varepsilon}^{r e f}(\mathbf{x}) d \Omega,}
$$

where $\varepsilon^{r e f}$ denotes the strain field obtained by the reference solution, and $\varepsilon^{C M C M}$ denotes the corresponding strain field obtained from CMCM. 


\subsection{Localized pressure on a $2 D$ fiber-reinforced structure}

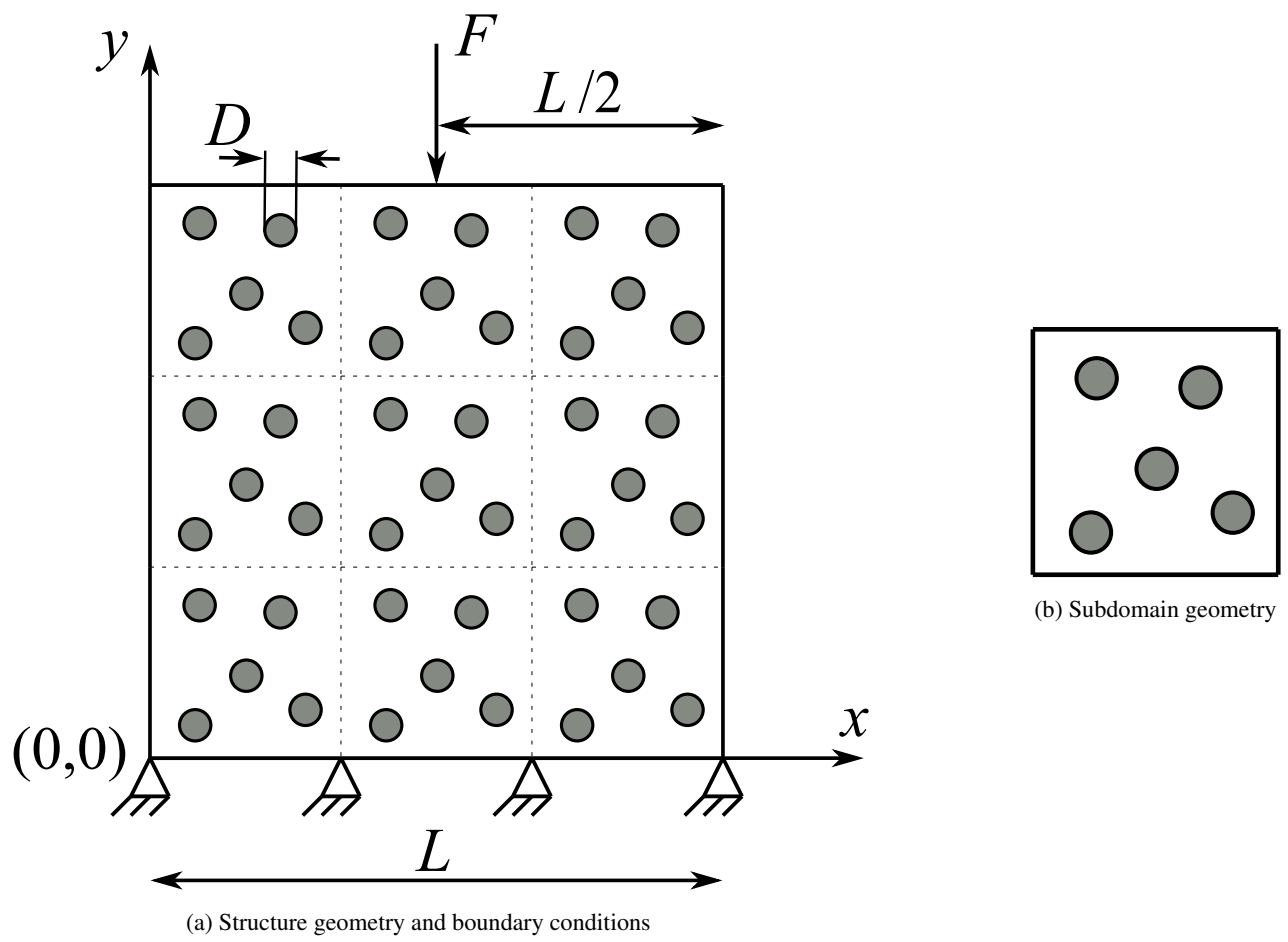

Figure 6: (a) Composite structure: geometry and boundary conditions; (b) subdomain used for off-line calculations.

In this first example, we consider a structure as depicted in Fig. 6a, subjected to a concentrated load. The length of the structure is $L=3 \mathrm{~mm}$ and all inclusions have the same diameter $D=0.01 \mathrm{~mm}$. The structure is decomposed into 9 periodic subdomains as described in Fig. 6b. The structure is subjected to a concentrated force located in one point $(x=L / 2, y=L)$. Since the structure is periodic, the subdomain problems defined in section 3.1 are calculated only for the geometry described in Fig. 6b. Fig 7 shows three different meshes used for the coarse mesh problem. The finite element mesh used for the reference DNS solution contains 49448 linear triangular elements, corresponding to 50050 dof. On the other hand, each subdomain mesh of the structure used for the CMCM method contains 5476 linear triangular elements corresponding to 5678 degrees of freedom per subdomain, which sums up to 51102 dof for the whole structure (9 subdomains). This is approximately the same number of dof as the reference structure, it is then appropriate to compare the solutions on these 2 meshes. The coarse meshes are composed of quadratic triangular elements, whose number of elements and number of dof are summarized in Table 3 .

\begin{tabular}{cccc}
\hline & Macro mesh 1 & Macro mesh 2 & Macro mesh 3 \\
\hline Element type & T6 & T6 & T6 \\
\hline Number of elements & 6 & 78 & 198 \\
\hline Number of dofs & 42 & 362 & 874 \\
\hline
\end{tabular}

Table 2: Number of elements and dofs for each coarse mesh used in calculations (T6 denotes quadratic triangular elements).

In this example, the coarse mesh 2 is refined in the entire structure while the coarse mesh 3 is only refined at the loading point. We can see in Fig. 8 that refining locally the coarse mesh near singularities or applied loads can gratefully increase the accuracy of the solution. We can see here an advantage of the extension proposed in the present work: such refinement is done without new subdomain calculation, then the computational increase remains low. Finally, here both non-periodic microstructure and non-conforming coarse meshes are employed, showing the capability of the method to work in such cases. 


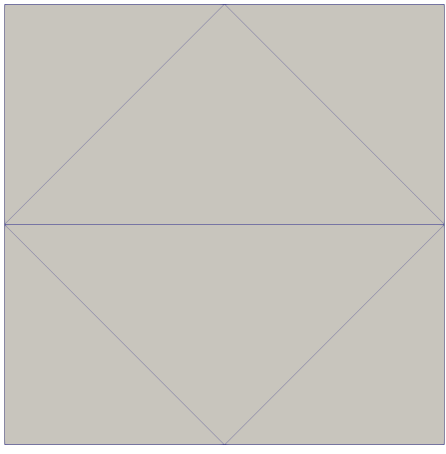

(a) Coarse mesh 1

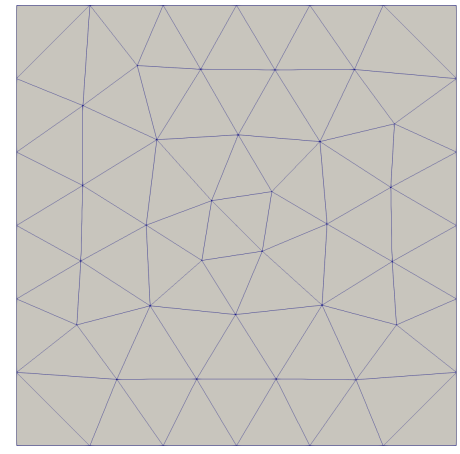

(b) Coarse mesh 2

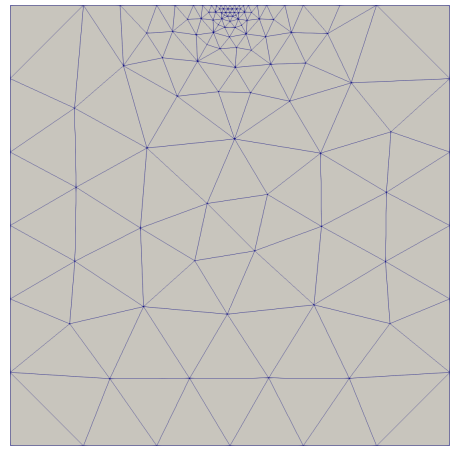

(c) Coarse mesh 3

Figure 7: Coarse mesh for solving the global structure problem.

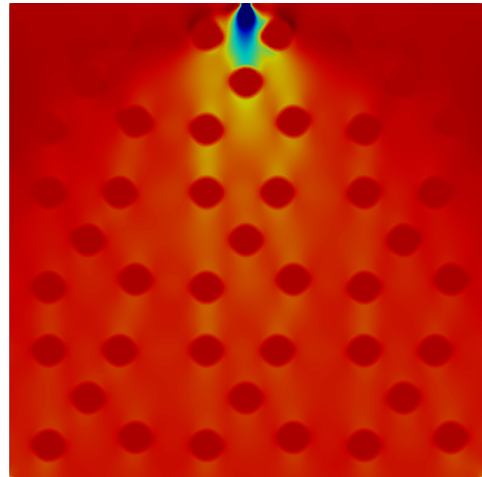

(a) Reference solution (DNS)

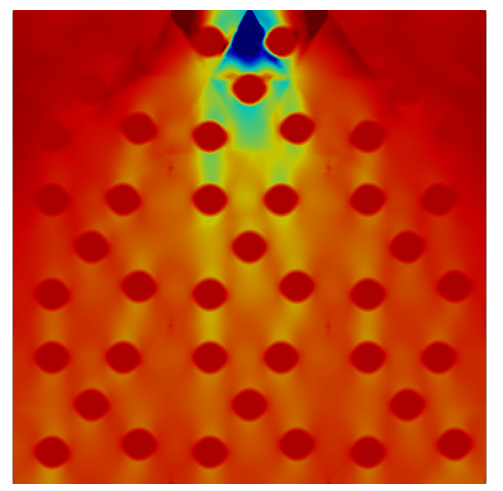

(c) Macro mesh 2

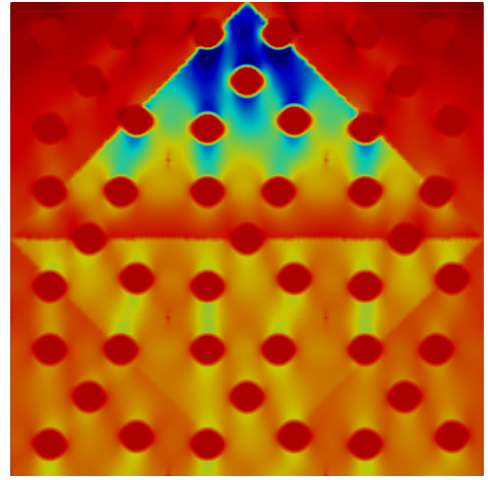

(b) Macro mesh 1

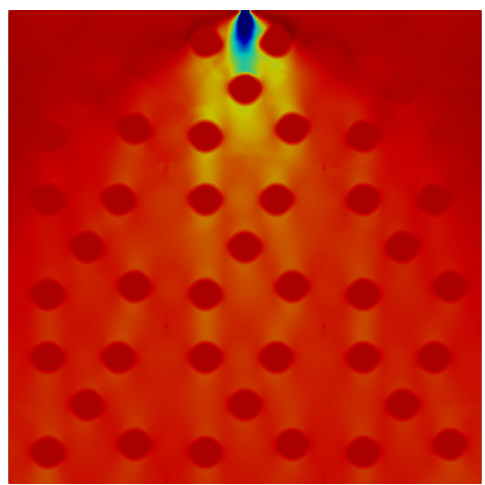

(d) Macro mesh 3 $\varepsilon_{22}(\mathbf{x})$

5.0e-02

$-0$

$-0.05$

$-0.1$

$-0.15$

$-0.2$

$-0.25$

$-0.3$

$-0.35$

$-0.4$

$-0.45$

$-5.0 e-01$

Figure 8: Relocalized strain field $\varepsilon_{22}(\mathbf{x})$ using the different coarse meshes. 


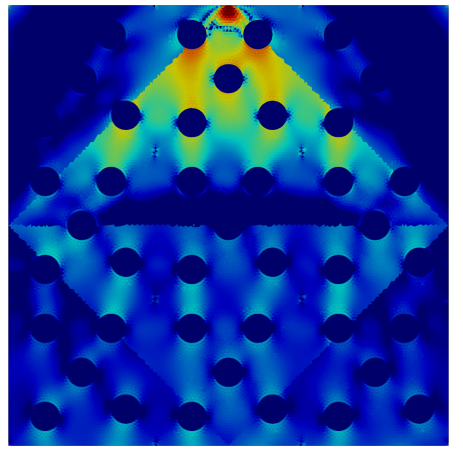

(a) Coarse mesh 1

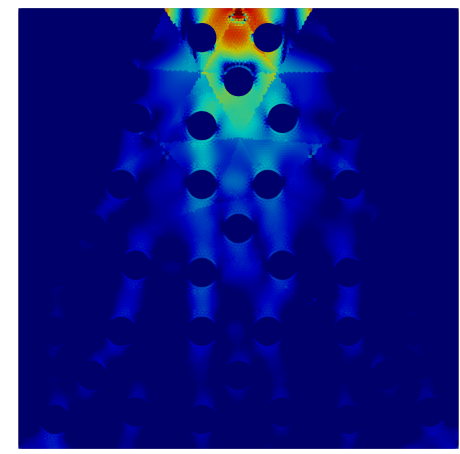

(b) Coarse mesh 2

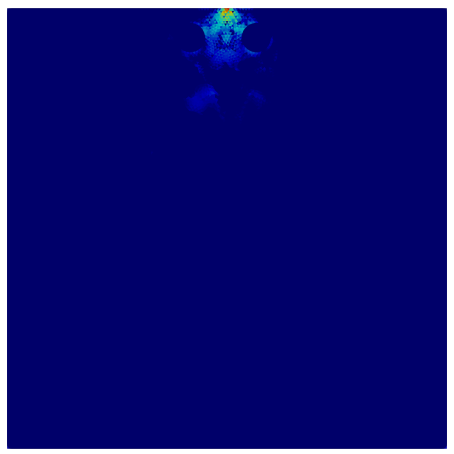

(c) Coarse mesh 3

$\begin{array}{lllllll}1.0 e-08 & 1 e-7 & 1 e-6 & 1 e-5 & 0.0001 & 0.001 & 1.0 e-02\end{array}$

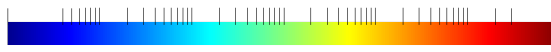

Figure 9: Local energy error distributions for the different used coarse meshes.

\section{2. $2 D$ random heterogeneous structure.}

In this example, we consider a randomly distributed reinforced-fiber square structure as depicted in Fig 10a. The size of the structure is $L=1 \mathrm{~mm}$. It is composed of 30 circular inclusions randomly distributed. The diameters of the inclusions are also random, ranging from 0.002 to $0.02 \mathrm{~mm}$. The structure is decomposed into 9 subdomains, as shown in Fig. 11a. To define these subdomains, the coordinates of the elements centers are tested as belonging to square domains. As the local mesh does not conform to regular square domains, the boundaries of the subdomains are not regular (see Fig. 11a). Since the microstructure is here not periodic, the subdomain problems need to be computed independently during off-line calculations. The computational cost for these calculations therefore increases, but as all the subdomain problems are independent, they can all be done in parallel, and the increase can in fact be null if sufficient number of cores are available. In this example, the extended subdomain technique described in section 3.1 is applied since the subdomain interfaces cut the inclusions. Figs 13 shows the evolution of the global error in the energy norm as a function of $\beta$ (see Eq. 9 ) for three cases of property contrast, using both consistent and approximated integration schemes presented in section 4 . We can see that when the mechanical contrast ratio $E_{i} / E_{m}$ is high, a high value of the error is obtained. However, increasing the value of $\beta$ decreases the global error. It is worth noting that in the case of 9 subdomains, the global error curves converge when $\beta=0.3$ for $E_{i} / E_{m}=10^{6}$, and $\beta=0.2$ for $E_{i} / E_{m}=10$ and $10^{3}$. In addition, it can be seen that using consistent integration scheme did reduce the global error in all three cases. However the gain is not significant and in view of the added complexity brought by the consistent integration. For this reason, we apply the approximated integration schemes in the next examples, and more specifically in the 3D examples. 


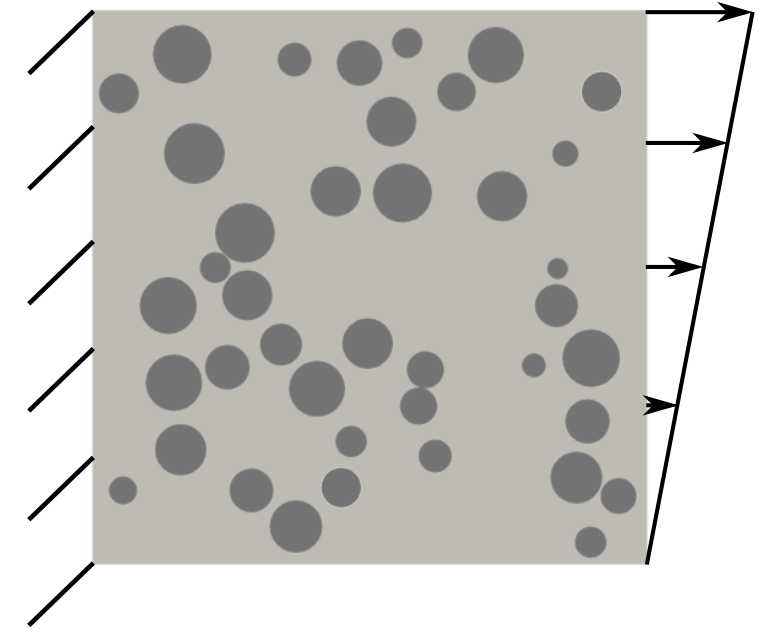

(a) Structure geometry and boundary conditions

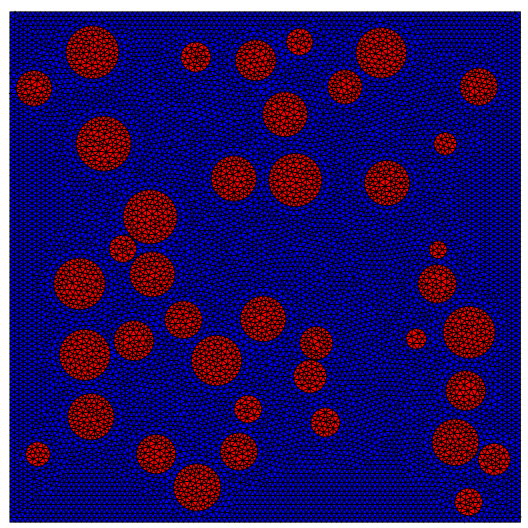

(b) Reference finite element mesh

Figure 10: Structure geometry, boundary conditions and finite element mesh.
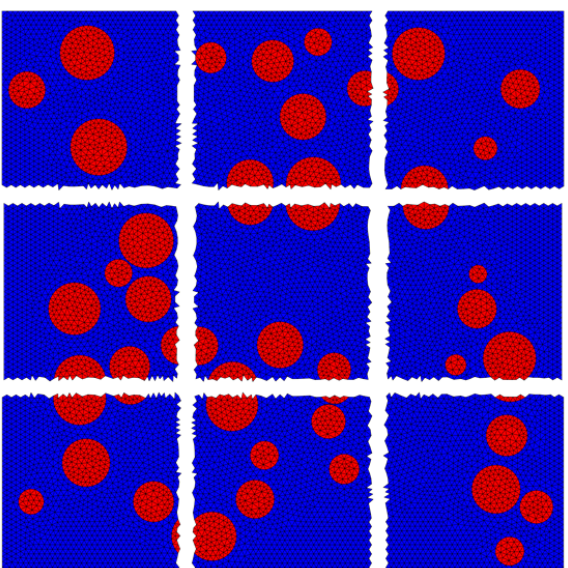

(a) Structure decomposed into 9 subdomains

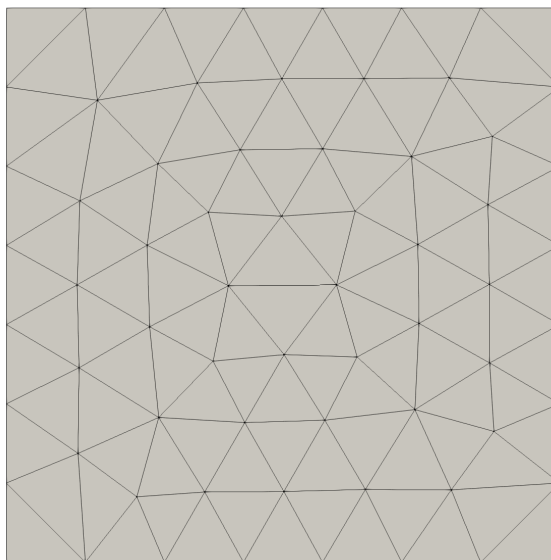

(b) Coarse mesh

Figure 11: Reference structure decomposed into 9 subdomains (left) and macro mesh used for global calculations (right). 


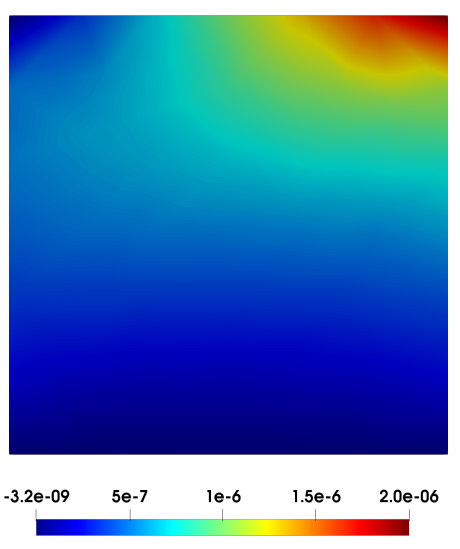

(a) $\varepsilon_{11}^{\text {reduced }}(\mathbf{x})$ using approximated integration scheme

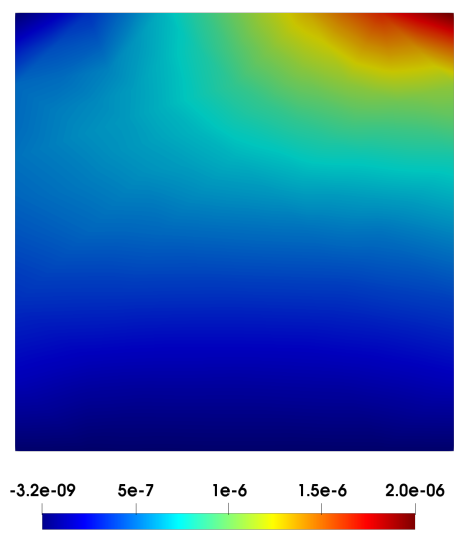

(b) $\varepsilon_{11}^{\text {exact }}(\mathbf{x})$ using consistent integration scheme

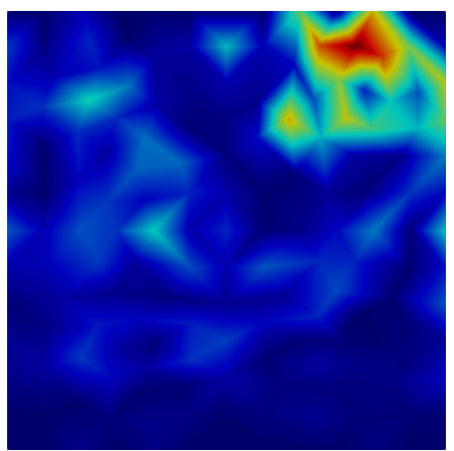

$2.7 e-252 e-9 \quad 4 e-9 \quad 6 e-9 \quad 8 e-9 \quad 1 e-8 \quad 1.2 e-8 \quad 1.5 e-08$

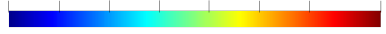

(c) $\left|\varepsilon_{11}^{\text {exact }}-\varepsilon_{11}^{\text {reduced }}\right|$

Figure 12: Macro strain field in the $x$ - direction using: (a) approximated integration scheme, (b) consistent integration scheme and (c) difference between these two fields.

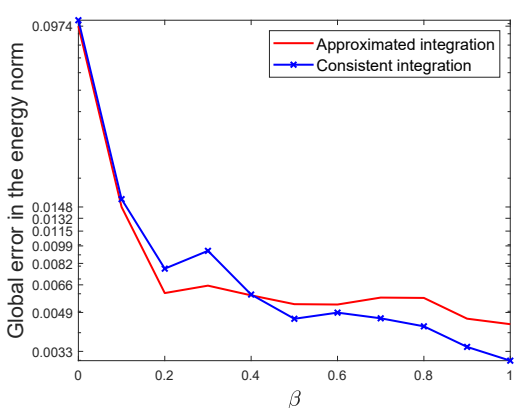

(a) $E_{i} / E_{m}=10$

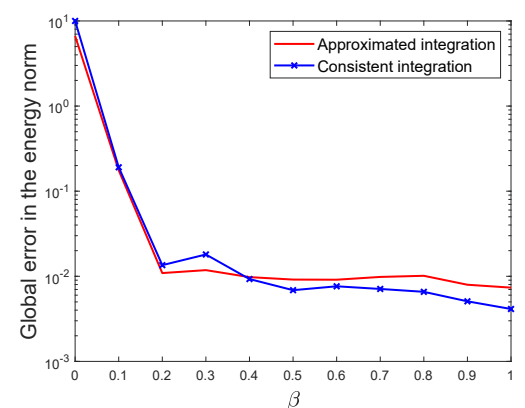

(b) $E_{i} / E_{m}=10^{3}$

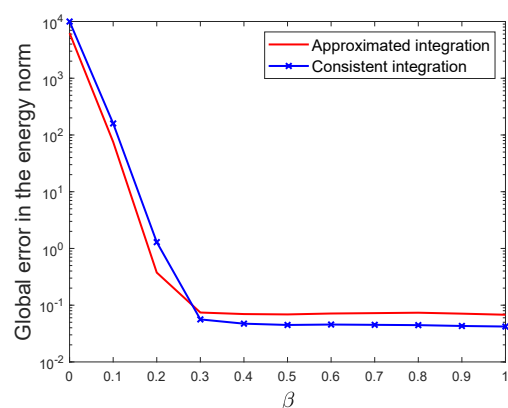

(c) $E_{i} / E_{m}=10^{6}$

Figure 13: Comparison of global error between approximated and consistent integration scheme in the case of $E_{i} / E_{m}=10,10^{3}$ and $10^{6}$ 


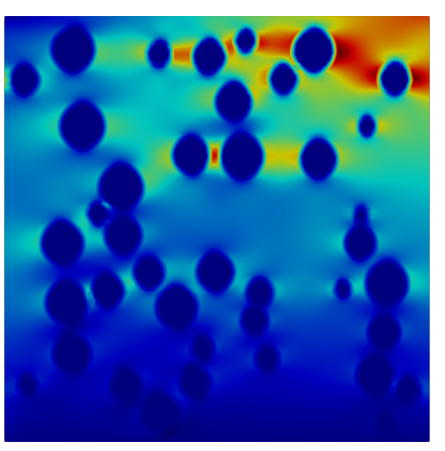

(a) Reference solution

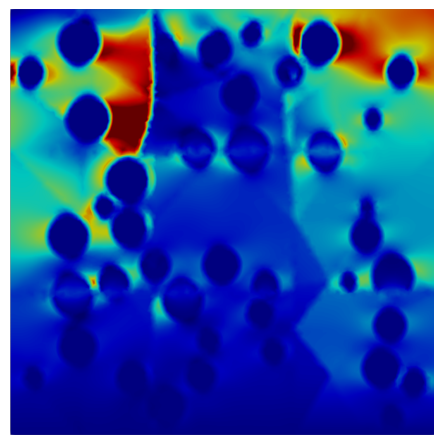

(b) CMCM solution for $\beta=0$

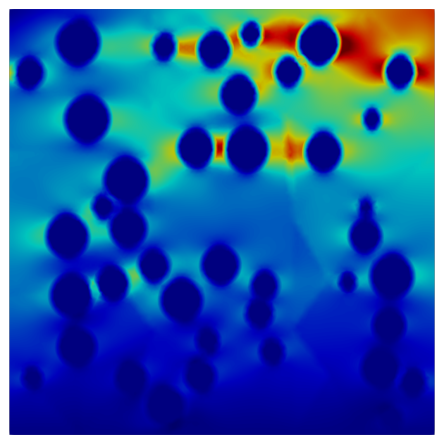

(c) CMCM solution for $\beta=0.3$

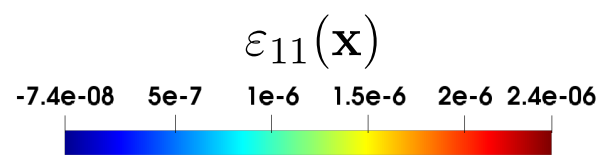

Figure 14: Strain fields $\varepsilon_{11}(\mathbf{x})$ : (a) reference solution, (b) relocalized CMCM solution for $\beta=0$ and (c) relocalized CMCM solution for $\beta=0.3$ and $E_{i} / E_{m}=10^{6}$.

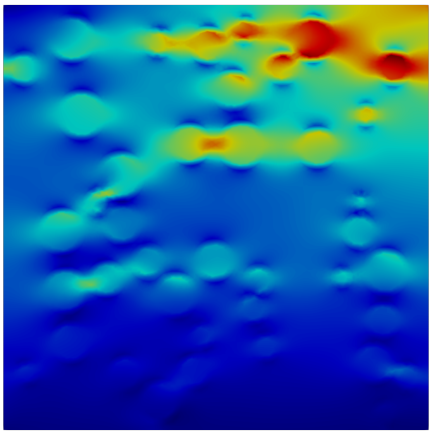

(a) Reference solution

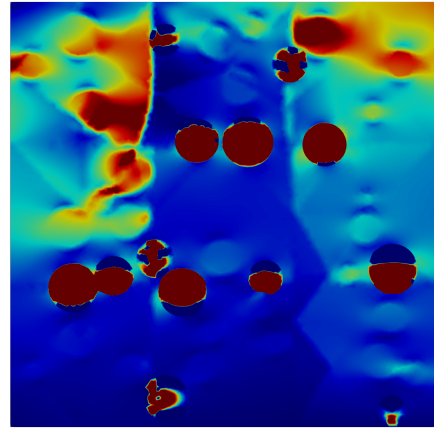

(b) CMCM solution with $\beta=0$

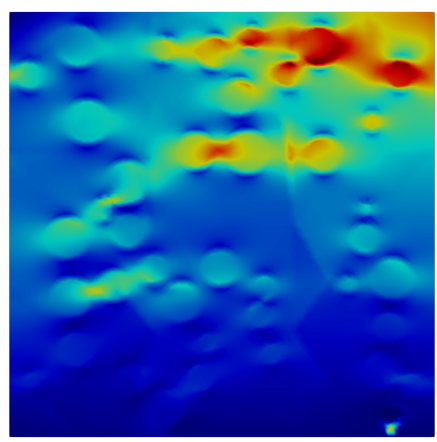

(c) $\mathrm{CMCM}$ solution with $\beta=0.3$

$$
\sigma_{11}(\mathbf{x})(\mathrm{MPa})
$$

$-6.3 e-08$

$2 e-6$

$4 e-6$

$6 e-6 \quad 8.0 e-06$

Figure 15: Stress fields $\sigma_{11}(\mathbf{x})(\mathrm{MPa})$ : (a) reference solution, (b) relocalized CMCM solution for $\beta=0$ and (c) relocalized CMCM solution for $\beta=0.3$ and $E_{i} / E_{m}=10^{6}$.

Figs. 16 show the evolution of global energy errors and online computational time as a function of the number of subdomains, respectively, for $E_{i} / E_{m}=10^{6}$. We can see that the global error slightly increases when more subdomains are used. However choosing a higher value of $\beta$, significantly reduce the errors. The increase of the number of subdomains decreases the computational time for the online (coarse mesh) calculations. 


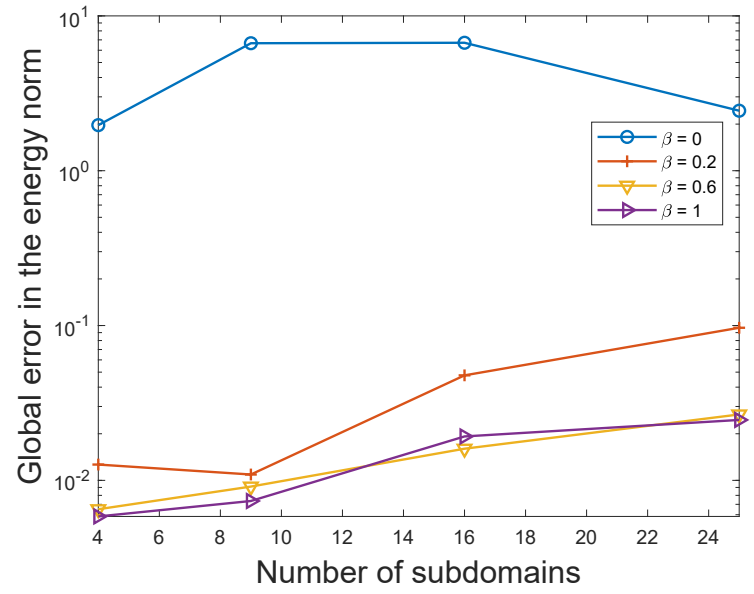

(a) Evolution of global error as a function of the number of subdomains

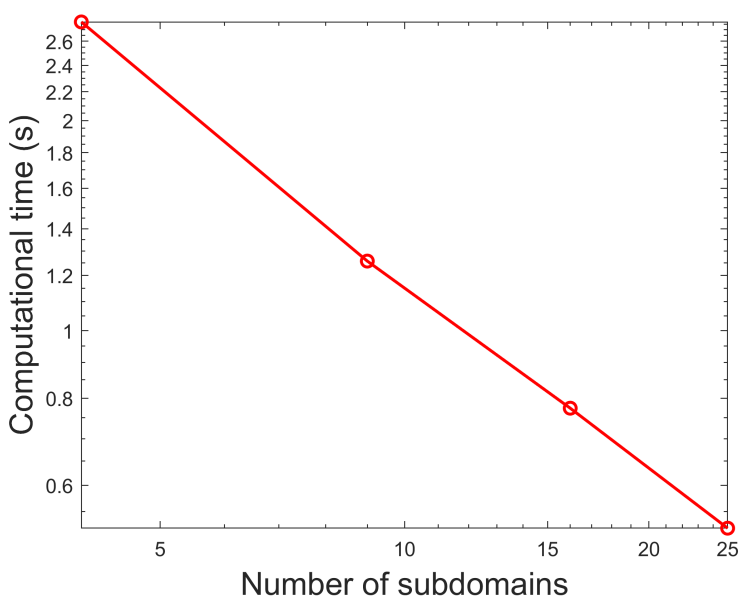

(b) Computational time during online calculations as function of number of subdomains

Figure 16: Evolution of error and computational time in function of number of subdomains for $E_{i} / E_{m}=10^{6}$.

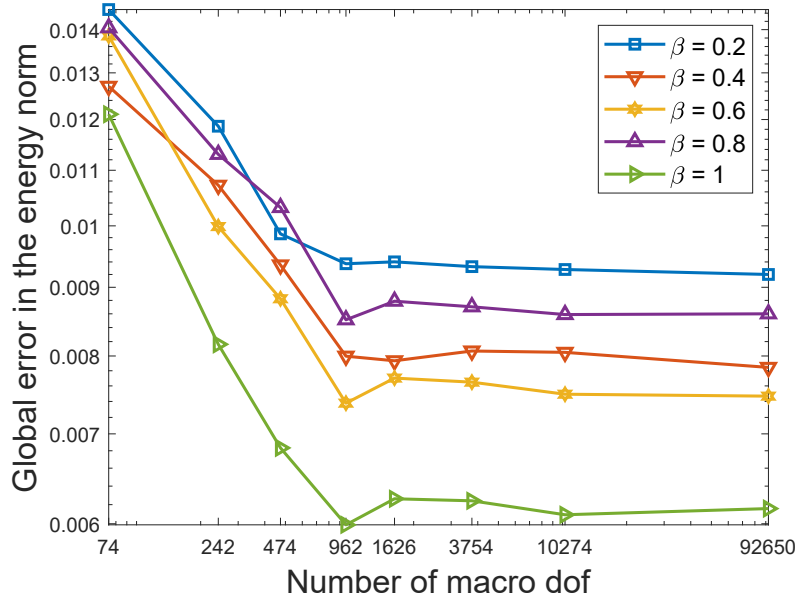

(a) Global energy error with respect to the number of dof in the macro mesh

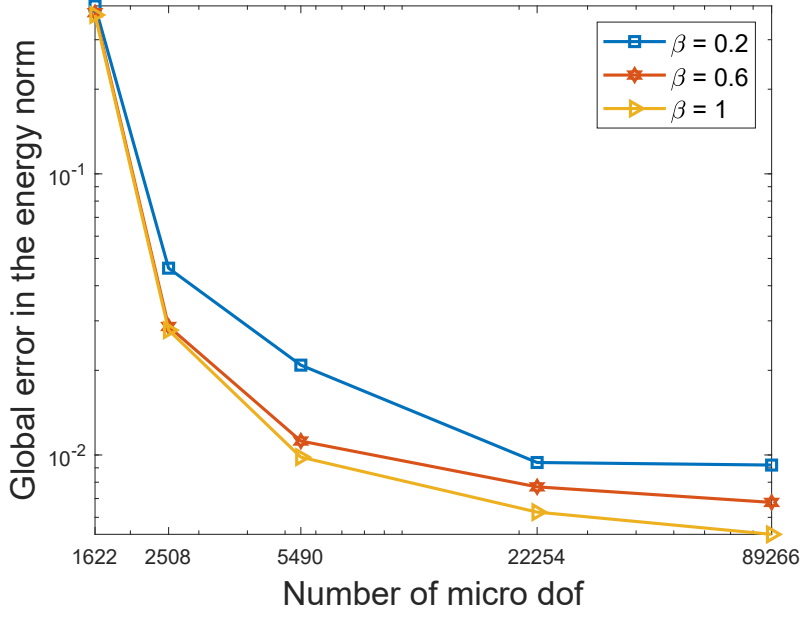

(b) Global energy error as a function of number of dof in the micro mesh

Figure 17: Evolution of global energy error with respect to the number of macro and micro dof for $E_{i} / E_{m}=10^{6}$.

Fig. 17 depicts the evolution of global energy error with respect to the number of macro and micro dof. It can be seen that the value of global error decreases when the number of both micro and macro mesh. However at a certain number of dof, the global error starts to converge, for example with a value of 982 in terms of macro dof and approximately 22254 in terms of mico dof.

From the above results, we can conclude that the present technique can be applied to non-periodic microstructures, with non-conforming coarse meshes.

\section{3. $3 D$ tomography of concrete}

In this example, the CMCM is applied to a structure characterized by micro tomography, including a large number of dof to show the potential of the method. The structure is a sample made of concrete (see Fig. 18). The sample contains 3 phases, sand grains, plaster matrix and voids. The properties of each phase are provided in Table 3 . To apply the proposed method, the structure is decomposed into 4096 subdomains as depicted in Fig. 19 The corresponding mesh for the whole structure contains 148,604,196 elements, corresponding to 448,351,500 dof. The coarse mesh 
contains 390,462 degrees of freedom. To limit computational costs, in this example we have only investigated two cases $\beta=0$ and $\beta=0.2$. In this example where all subdomains are different, the computational times related to off-line calculations are quite expensive. However, once off-line calculations have been conducted, the coarse mesh problem can be solved for several loading cases at low computational costs. In this example, we have tested 3 types of loading: traction, bending and concentrated load, as depicted in Fig. 20

\begin{tabular}{cccc}
\hline & Grains of sand & Matrix & Voids \\
\hline Young's modulus (MPa) & 5 & 1 & $10^{-6}$ \\
\hline Poisson coefficient & 0.3 & 0.3 & 0 \\
\hline
\end{tabular}

Table 3: Material properties of concrete.

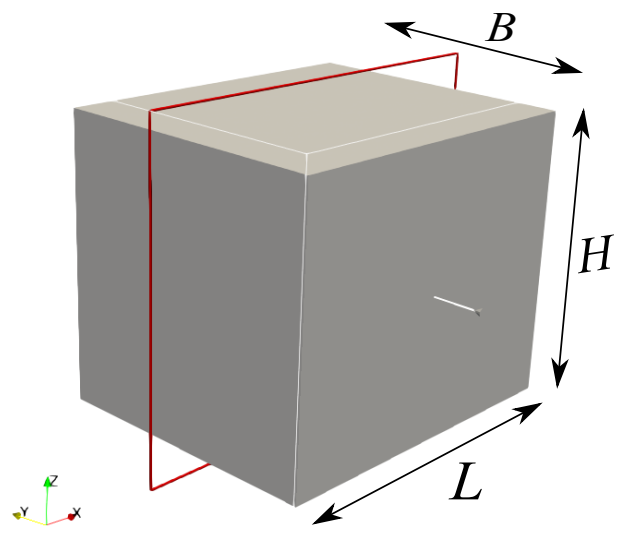

(a) Structure geometry

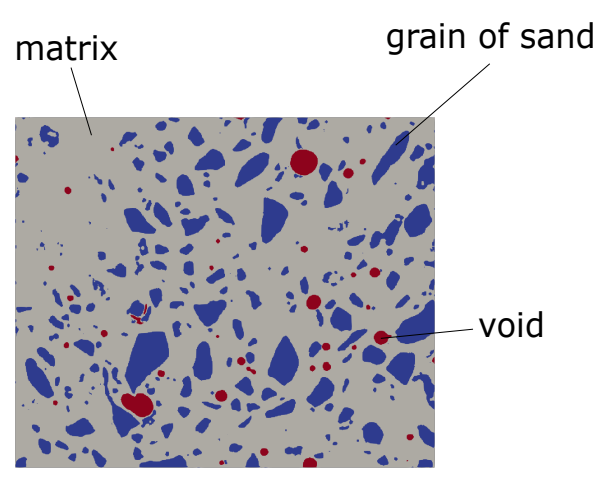

(b) Cross section in the plane $y=B / 2$

Figure 18: Structure geometry and its cross section.

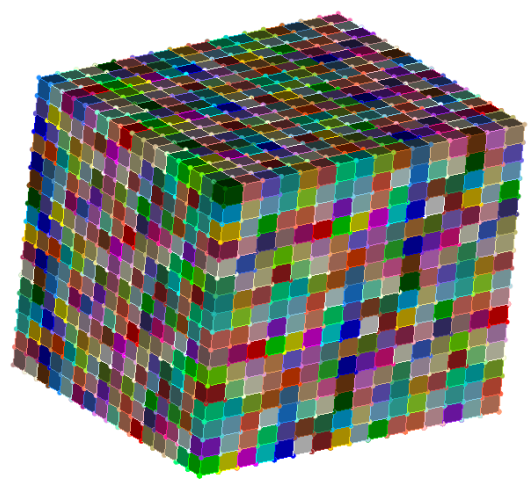

(a) Structure decomposed into $16 \times 16 \times 16$ subdomains.

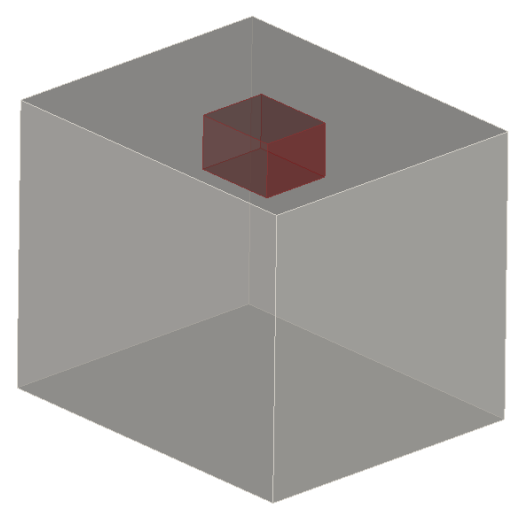

(b) A block used for relocalization and post-treatment

Figure 19: Decomposition of the microstructure (a) and the block used for local field comparison (b). 


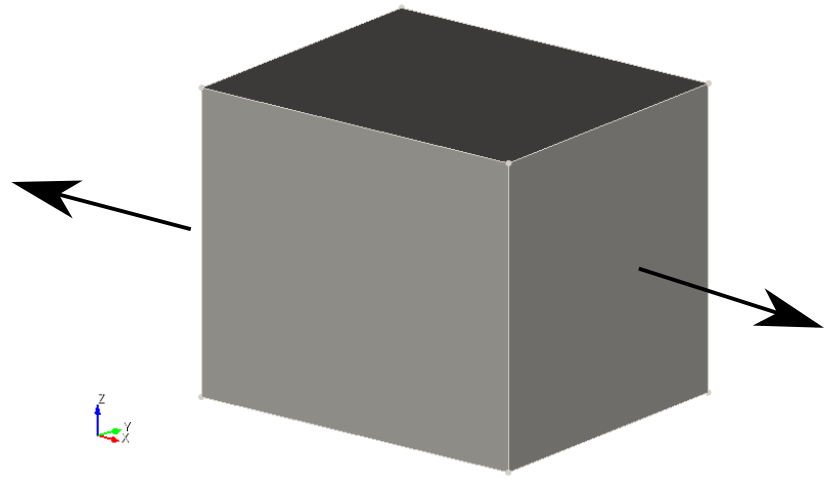

(a) Traction boundary conditions

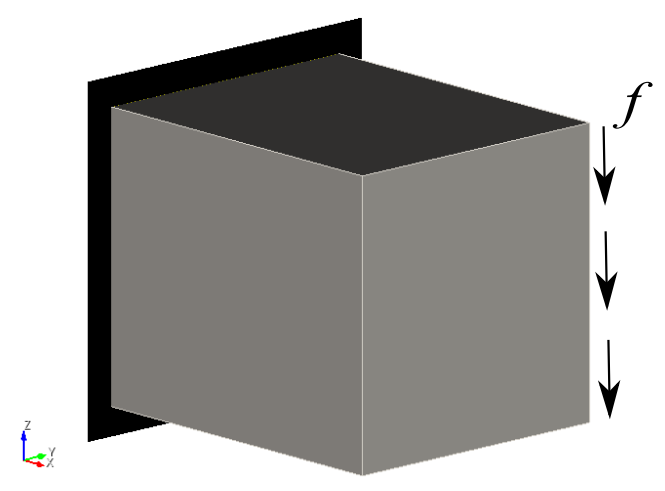

(c) Bending boundary conditions

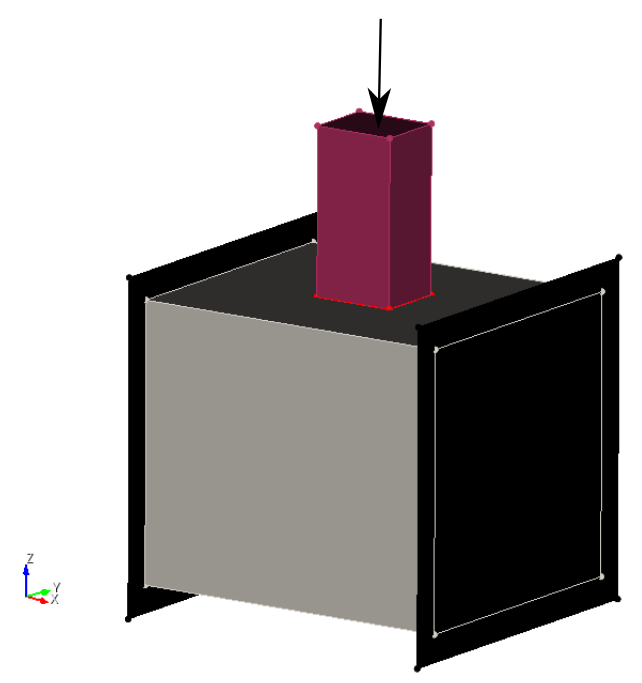

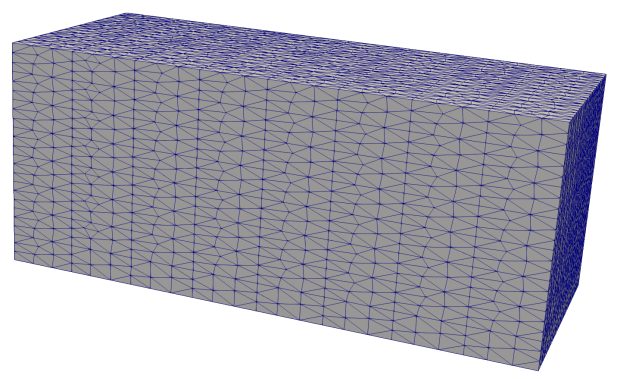

(b) Traction deformed shape

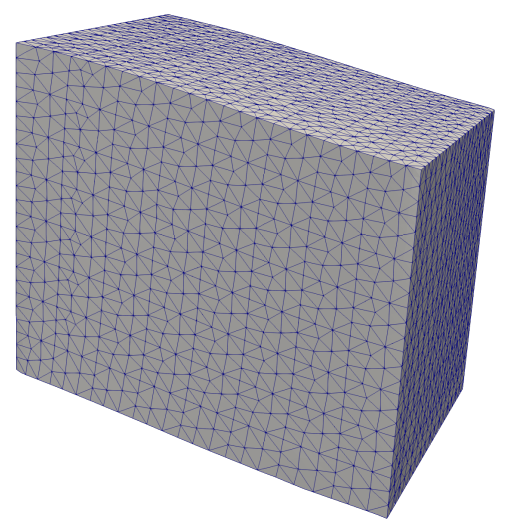

(d) Bending deformed shape

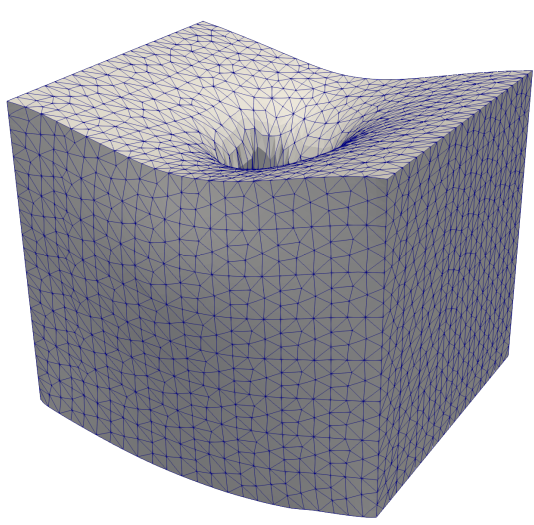

(f) Concentrated load's deformed shape

(e) Concentrated load

Figure 20: Three loading types of the structure and their corresponding deformed shapes. 


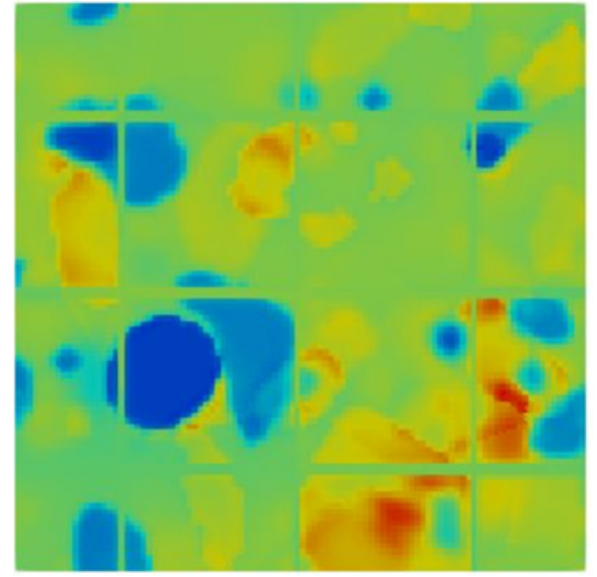

(a) $\beta=0$

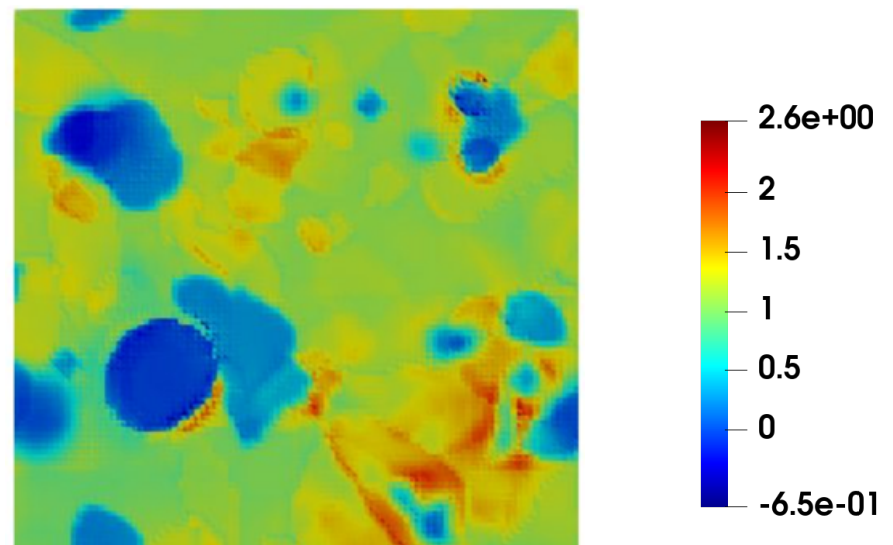

(b) $\beta=0.2$

Figure 21: Relocalized strain solution in a cross section of the relocalization block for (a) $\beta=0$ and (b) $\beta=0.2$

From Fig 21, it can be seen that when $\beta=0$, discontinuities are significantly induced at the interfaces of the subdomains. However when $\beta=0.2$, these discontinuities are reduced. In the next figures, only solutions of the problem with $\beta=0.2$ are shown in order to avoid multiplying the number of figures.

From the obtained results, it can be seen that in all three cases, the relocalized strain and stress fields have well captured the effects of three loadings types on the microstructure. In addition, even with a small value of $\beta$, we were able to reduce significantly the discontinuities at subdomain interfaces. 


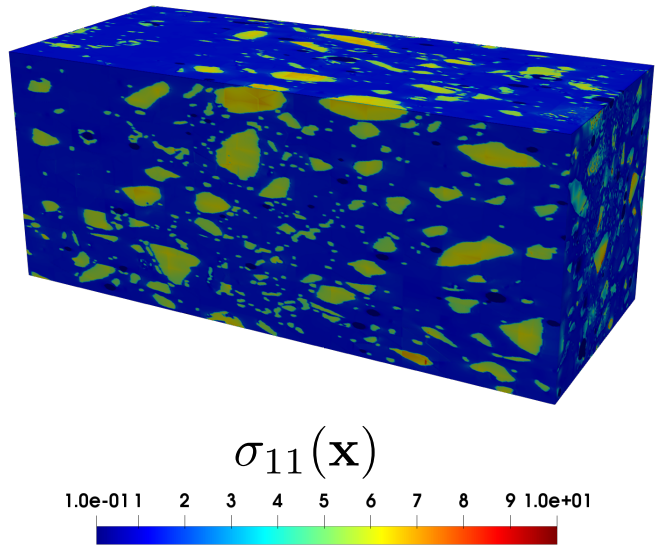

(a) Traction's relocalized 11-stress field
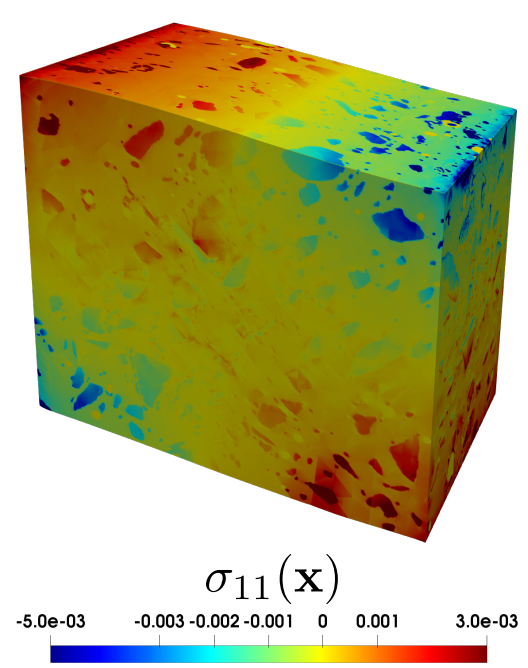

(c) Bending's relocalized 11-stress field

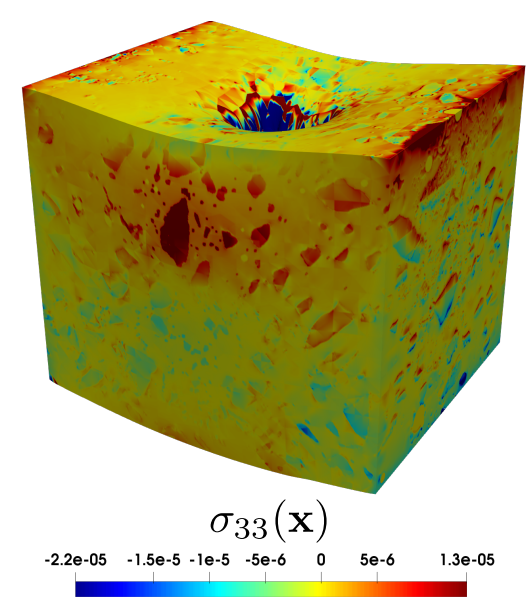

(e) Concentrated load's relocalized 33-stress field

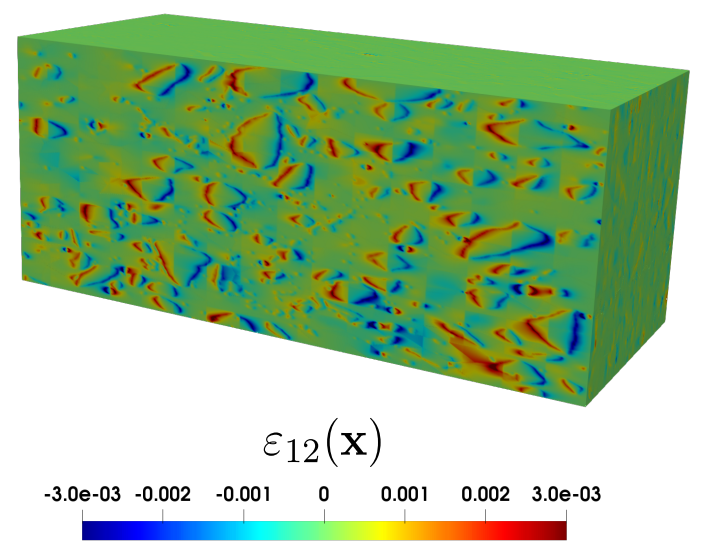

(b) Traction's relocalized 12-strain field

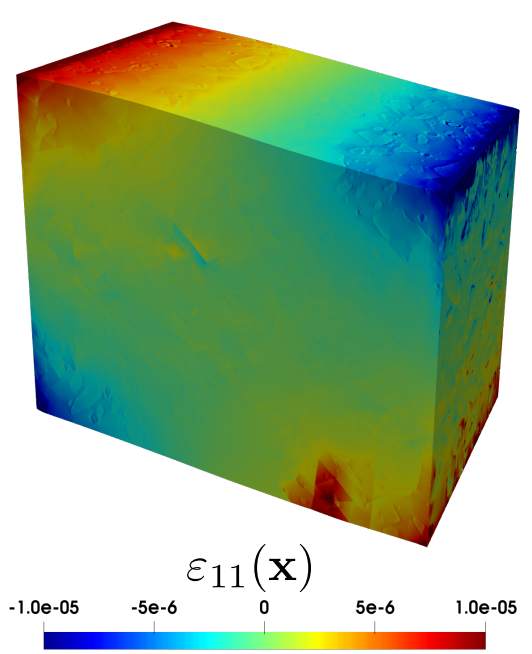

(d) Bending's relocalized 11-strain field

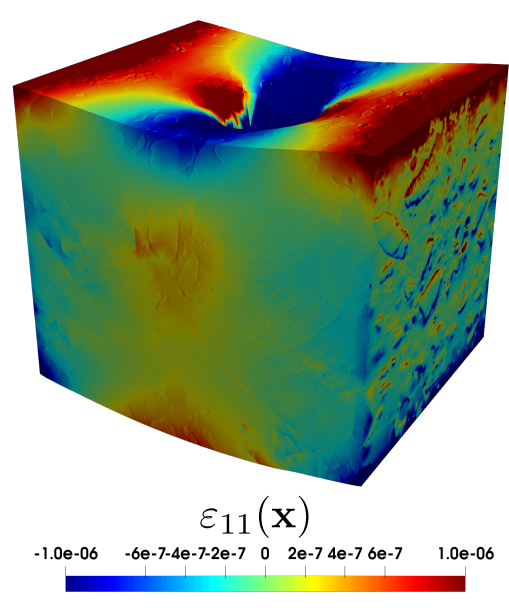

(f) Concentrated load's relocalized 11-strain field

Figure 22: Relocalized stress and strain fields for 3 considered cases. 


\section{Conclusion}

A Coarse Mesh Condensation Multiscale (CMCM) method, initially introduced in [47] for solving periodic composite structures, has been extended in this work to perform direct numerical simulations (DNS) of large, non-periodic heterogeneous structures which may arise e.g. from micro tomography images of heterogeneous structures. The main idea of the method is to use a reduced representation of the subdomains boundary displacements, which can be used to condense the solutions in the subdomains on their boundaries. Then, the reduced degrees of freedom (dof) are expressed with respect to the dof of a mapping coarse mesh on the whole structure. As a result, the problem is solved on the coarse mesh dof only. The first novelty introduced in this paper is the use of non-periodic subdomains which allows decomposing arbitrary, non-periodic heterogeneous structures. The second novelty is the use of a nonconforming coarse mesh. Then, it is no more required that the coarse mesh conforms with the subdomain boundaries. A numerical analysis has been performed on benchmarks. It has been shown that when heterogeneities cross the boundaries of the subdomains, discontinuities can occur in the reconstructed local solutions. Use of extended subdomains is employed to drastically reduce the errors in that case. We have shown that using a non-periodic coarse mesh allows reducing the errors in the reconstructed solution by local refinement around singularities such as concentrated loads. Finally, we have shown that this method can be used to perform DNS on micro tomography models of heterogeneous structures directly involving hundred of millions of dof on a standard computer.

Another possible application of the method lies in the analysis of full-field "virtual" mesoscopic composite structures obtained e.g. from direct simulation of forming processes (see e.g. [53]). The resulting geometry of such models is seldom used as the initial topology of a subsequent structure problem, chiefly because of its computing cost in implicit simulations, even if a simple coupon were to be considered [47]. Further, the non-periodic nature of the formed microstructure also prevents an efficient use of periodic homogenization-based methods. Then, the CMCM variant proposed hereafter is also designed with these use cases in mind, specifically so that they may become tractable in the very near future. Even though challenging, another perspective for this work is its extension to nonlinear problems, by applying the present framework to the linearized problem involved in an iterative Newton-like solving procedure.

\section{Appendix A. Reconstruction of the local strain and displacement fields.}

In this section, we specify the reconstruction process for local displacement fields. We denote by $\mathbf{x}^{\prime}$ a local coordinate system related to one subdomain. For example, in the case where each subdomain is associated with a periodic unit cell, the local coordinate system might include an origin at the center of the unit cell. The displacement field is the global coordinate system $\mathbf{x}$ is expressed as:

$$
\mathbf{u}(\mathbf{x})=\overline{\boldsymbol{\varepsilon}} \cdot \mathbf{x}+\frac{1}{2} \overline{\mathcal{A}}: \mathbf{x} \otimes \mathbf{x}+\tilde{\mathbf{u}}(\mathbf{x}),
$$

where $\tilde{\mathbf{u}}(\mathbf{x})$ and $\tilde{\boldsymbol{\varepsilon}}(\mathbf{x})$ denotes the fluctuations of the local displacement, which are non zero in subdomains containing heterogeneities. The localization problem being solved in the local coordinate system attached to the subdomain, we have:

$$
\mathbf{u}\left(\mathbf{x}^{\prime}\right)=\mathbf{U}^{\alpha}\left(\mathbf{x}^{\prime}\right) \overline{\mathbf{g}}^{\alpha} \quad \forall \mathbf{x}^{\prime} \in \Omega^{\alpha},
$$

where $\mathbf{u}^{1}\left(\mathbf{x}^{\prime}\right), \mathbf{U}^{\alpha}\left(\mathbf{x}^{\prime}\right)=\left[\mathbf{u}^{1}\left(\mathbf{x}^{\prime}\right), \mathbf{u}^{2}\left(\mathbf{x}^{\prime}\right), \ldots, \mathbf{u}^{N_{g}}\left(\mathbf{x}^{\prime}\right)\right]$, where $\mathbf{u}^{1}\left(\mathbf{x}^{\prime}\right), \mathbf{u}^{2}\left(\mathbf{x}^{\prime}\right), \ldots, \mathbf{u}^{N_{g}}\left(\mathbf{x}^{\prime}\right)$ are solutions of problem (5)-(6) in the subdomain $\Omega^{\alpha}$.

From (A.1) and A.2) we have:

$$
\tilde{\mathbf{u}}\left(\mathbf{x}^{\prime}\right)=\mathbf{U}^{\alpha}\left(\mathbf{x}^{\prime}\right) \overline{\mathbf{g}}^{\alpha}-\overline{\boldsymbol{\varepsilon}} \cdot \mathbf{x}^{\prime}-\frac{1}{2} \overline{\mathcal{A}}: \mathbf{x}^{\prime} \otimes \mathbf{x}^{\prime} \quad \forall \mathbf{x}^{\prime} \in \Omega^{\alpha},
$$

Substituting (A.3) in A.1) we obtain:

$$
\begin{aligned}
\mathbf{u}(\mathbf{x}) & =\overline{\boldsymbol{\varepsilon}} \cdot \mathbf{x}+\frac{1}{2} \overline{\mathcal{A}}: \mathbf{x} \otimes \mathbf{x}+\mathbf{U}^{\alpha}\left(\mathbf{x}^{\prime}\right) \overline{\mathbf{g}}^{\alpha}-\overline{\boldsymbol{\varepsilon}} \cdot \mathbf{x}^{\prime}-\frac{1}{2} \overline{\mathcal{A}}: \mathbf{x}^{\prime} \otimes \mathbf{x}^{\prime}, \\
& =\mathbf{U}^{\alpha}\left(\mathbf{x}^{\prime}\right) \overline{\mathbf{g}}^{\alpha}+\overline{\boldsymbol{\varepsilon}} \cdot\left(\mathbf{x}-\mathbf{x}^{\prime}\right)+\frac{1}{2} \overline{\mathcal{A}}:\left(\mathbf{x} \otimes \mathbf{x}-\mathbf{x}^{\prime} \otimes \mathbf{x}^{\prime}\right) .
\end{aligned}
$$




\section{References}

[1] V. Kouznetsova, M. Geers, W. Brekelmans, Multi-scale constitutive modeling of heterogeneous materials with gradient enhanced computational homogenization scheme, International Journal for Numerical Methods in Engineering 54 (2002) 1235-1260.

[2] V. Kouznetsova, M. Geers, W. Brekelmans, Multi-scale second order computational homogenization of multi-phase materials: a nested finite element solution strategy, Computer Methods in Applied Mechanics and Engineering 193 (2004) 5525-5550.

[3] X. Yuan, Y. Tomita, A micromechanical approach of nonlocal modeling for media with periodic microstructures, Mechanics Reasearch Communications 35 (2008) 126133.

[4] F. Bouyge, I. Jasiuk, M. Ostoja-Starzewski, A micromechanically based couple-stress model of an elastic two-phase composite, International Journal of Solids and Structures 38 (10-13) (2001) 1721-1735.

[5] F. Feyel, A multilevel finite element method $\left(\mathrm{FE}^{2}\right)$ to describe the response of highly non-linear structures using generalized continua, Computer Methods in Applied Mechanics and Engineering 192 (28-30) (2003) 3233-3244.

[6] S. Forest, K. Sab, Cosserat overall modelling of heterogeneous materials, Mechanics Research Communications 25(4) (1998) 449-454.

[7] T.-H. Tran, V. Monchiet, G. Bonnet, A micromechanics-based approach for the derivation of constitutive elastic coefficients of strain-gradient media, International Journal of Solids and Structures 49 (2012) 783-792.

[8] A. Tognevi, M. Guerich, J. Yvonnet, A multi-scale modeling method for heterogeneous structures without scale separation using a filter-based homogenization scheme, International Journal for Numerical Methods in Engineering 108(1-5) (2016) 3-25.

[9] T. Hui, C. Oskay, A nonlocal homogenization model for wave dispersion in dissipative composite materials, International Journal of Solids and Structures 50 (2013) 38-48.

[10] J. Fish, S. Kuznetsov, Computational continua, International Journal for Numerical Methods in Engineering 84 (7) (2010) 774-802.

[11] J. Fish, V. Filonova, D. Fafalis, Computational continua revisited, Computer Methods in Applied Mechanics and Engineering 102 (2015) $332-378$.

[12] J. Fish, P. Nayak, M. H. Holmes, Microscale reduction error indicators and estimators for a periodic heterogeneous medium, Computational Mechanics 14 (4) (1994) 323-338.

[13] C. Farhat, F.-X. Roux, A method of finite element tearing and interconnecting and its parallel solution algorithm, International Journal for Numerical Methods in Engineering 32 (6) (1991) 1205-1227.

[14] P. Le Tallec, Y.-H. De Roeck, M. Vidrascu, Domain decomposition methods for large linearly elliptic three-dimensional problems, Journal of Computational and Applied Mathematics 34 (1) (1991) 93-117.

[15] D. J. Rixen, C. Farhat, A simple and efficient extension of a class of substructure based preconditioners to heterogeneous structural mechanics problems, International Journal for Numerical Methods in Engineering 44 (4) (1999) 489-516.

[16] P. Gosselet, C. Rey, D. J. Rixen, On the initial estimate of interface forces in FETI methods, Computer Methods in Applied Mechanics and Engineering 192 (25) (2003) 2749-2764.

[17] N. Spillane, V. Dolean, P. Hauret, F. Nataf, D. Rixen, Solving generalized eigenvalue problems on the interfaces to build a robust two level FETI method, International Journal for Numerical Methods in Engineering (2012) (2012)

[18] P. Gosselet, D. Rixen, F.-X. Roux, N. Spillane, Simultaneous FETI and block FETI: Robust domain decomposition with multiple search directions, International Journal for Numerical Methods in Engineering 104 (10) (2015) 905-927.

[19] N. Spillane, An adaptive multipreconditioned conjugate gradient algorithm, SIAM Journal on Scientific Computing 38 (3) (2016) A1896A1918.

[20] C. Bovet, A. Parret-Fréaud, N. Spillane, P. Gosselet, Adaptive multipreconditioned FETI: scalability results and robustness assessment, Computers \& Structures 193 (2017) 1-20.

[21] P. Ladevèze, O. Loiseau, D. Dureisseix, A micro-macro and parallel computational strategy for highly heterogeneous structures, International Journal for Numerical Methods in Engineering 52 (1-2) (2001) 121-138.

[22] P. Ladevèze, J.-C. Passieux, D. Néron, The latin multiscale computational method and the proper generalized decomposition, Computer Methods in Applied Mechanics and Engineering 199 (21-22) (2010) 1287-1296.

[23] K. Stüben, Algebraic multigrid (AMG): experiences and comparisons, Applied mathematics and computation 13 (3-4) (1983) 419-451.

[24] J. Ruge, K. Stüben, Efficient solution of finite difference and finite element equations by algebraic multigrid AMG, Gesellschaft f. Mathematik u. Datenverarbeitung, 1984

[25] J. W. Ruge, K. Stüben, Algebraic multigrid, in: Multigrid methods, SIAM, 1987, pp. 73-130.

[26] K. Stüben, A review of algebraic multigrid, in: Numerical Analysis: Historical Developments in the 20th Century, Elsevier, 2001, pp. 331-359.

[27] T. I. Zohdi, P. Wriggers, A domain decomposition method for bodies with heterogeneous microstructure basedon material regularization, International Journal of Solids and Structures 36 (17) (1999) 2507-2525.

[28] T. I. Zohdi, P. Wriggers, C. Huet, A method of substructuring large-scale computational micromechanical problems, Computer Methods in Applied Mechanics and Engineering 190 (43-44) (2001) 5639-5656.

[29] M. Hautefeuille, J.-B. Colliat, A. Ibrahimbegovic, H. Matthies, P. Villon, A multi-scale approach to model localized failure with softening, Computers \& Structures 94 (2012) 83-95.

[30] A. Huerta, E. Nadal, F. Chinesta, Proper generalized decomposition solutions within a domain decomposition strategy, International Journal for Numerical Methods in Engineering 113 (13) (2018) 1972-1994.

[31] E. Weinan, B. Engquist, X. Li, W. Ren, E. Vanden-Eijnden, Heterogeneous multiscale methods: a review, Commun. Comput. Phys 2 (3) (2007) 367-450.

[32] A. Abdulle, E. Weinan, B. Engquist, E. Vanden-Eijnden, The heterogeneous multiscale method, Acta Numerica 21 (2012) 1-87.

[33] T. Y. Hou, X.-H. Wu, A multiscale finite element method for elliptic problems in composite materials and porous media, Journal of computational physics 134 (1) (1997) 169-189.

[34] Y. Efendiev, T. Y. Hou, Multiscale finite element methods: theory and applications, Vol. 4, Springer Science \& Business Media, 2009. 
[35] H. B. Dhia, G. Rateau, The arlequin method as a flexible engineering design tool, International journal for numerical methods in engineering 62 (11) (2005) 1442-1462.

[36] F. Kelley, Mesh requirements for the analysis of a stress concentration by the specified boundary displacement method, in: Proceedings of the second international computers in engineering conference, ASME, 1982, pp. 39-42.

[37] F. Daghia, P. Ladevèze, A micro-meso computational strategy for the prediction of the damage and failure of laminates, Composite structures 94 (12) (2012) 3644-3653.

[38] J. Bénézech, G. Couégnat, Variational segmentation of textile composite preforms from x-ray computed tomography, Composite Structures 230 (2019) 111496

[39] M. Wangermez, O. Allix, P.-A. Guidault, O. Ciobanu, C. Rey, Interface coupling method for the global-local analysis of heterogeneous models: A second-order homogenization-based strategy, Computer Methods in Applied Mechanics and Engineering 365 (2020) 113032.

[40] J. Fish, V. Belsky, Multi-grid method for periodic heterogeneous media part 2: Multiscale modeling and quality control in multidimensional case, Computer Methods in Applied Mechanics and Engineering 126 (1-2) (1995) 17-38.

[41] J. Fish, V. Belsky, Multigrid method for periodic heterogeneous media part 1: Convergence studies for one-dimensional case, Computer Methods in Applied Mechanics and Engineering 126 (1) (1995) 1-16.

[42] C. Miehe, C. Bayreuther, On multiscale fe analyses of heterogeneous structures: from homogenization to multigrid solvers, International Journal for Numerical Methods in Engineering 71 (10) (2007) 1135-1180.

[43] N. Neuss, W. Jäger, G. Wittum, Homogenization and multigrid, Computing 66 (1) (2001) 1-26.

[44] H. Moulinec, P. Suquet, A numerical method for computing the overall response of nonlinear composites with complex microstructure, Computer Methods in Applied Mechanics and Engineering 157 (1998) 69-94.

[45] Y. Chen, L. Gélébart, C. Chateau, M. Bornert, C. Sauder, A. King, Analysis of the damage initiation in a sic/sic composite tube from a direct comparison between large-scale numerical simulation and synchrotron X-ray micro-computed tomography, International Journal of Solids and Structures 161 (2019) 111-126.

[46] A. Prakash, R. Lebensohn, Simulation of micromechanical behavior of polycrystals: finite elements versus fast fourier transforms, Modelling and Simulation in Materials Science and Engineering 17 (6) (2009) 064010.

[47] M. V. Le, J. Yvonnet, N. Feld, F. Detrez, The coarse mesh condensation multiscale method for parallel computation of heterogeneous linear structures without scale separation, Computer Methods in Applied Mechanics and Engineering 363 (2020) 112877.

[48] M. Abràmoff, P. Magalhães, S. Ram, Image processing with ImageJ, Biophotonics international 11 (7) (2004) 36-42.

[49] J. Bielak, O. G. E. Kim, Parallel octree-based finite element method for large-scale earthquake ground motion simulation, CCMES-Comp. Model. Eng. 10 (2) (2005) 99.

[50] R. G. S.H., Hollister, G. Charras, The accuracy of digital image-based finite element models, J. Biomed. Eng. 120 (1998) $289-295$.

[51] T. Nguyen, J. Yvonnet, M. Bornert, C. Chateau, F. Bilteryst, E. Steib, Large-scale simulations of quasi-brittle microcracking in realistic highly heterogeneous microstructures obtained from micro ct imaging, Extreme mechanics letters 17 (2017) 50-55.

[52] J. Yvonnet, Computational Homogenization of Heterogeneous Materials with Finite Elements, Springer Nature, 2019.

[53] D. Wang, N. Naouar, E. Vidal-Sallé, P. Boisse, Longitudinal compression and poisson ratio of fiber yarns in meso-scale finite element modeling of composite reinforcements, Composites Part B: Engineering 141 (12 2017). doi:10.1016/j.compositesb.2017.12.042 